\title{
Constrained Data-driven Optimal Iterative Learning Control
}

\author{
Ronghu Chi ${ }^{\mathrm{a}}$, Xiaohe Liu ${ }^{\mathrm{a}}$, Ruikun Zhang ${ }^{\mathrm{b}}$, Zhongsheng Hou ${ }^{\mathrm{c}}$, Biao Huang ${ }^{\mathrm{d}}$ \\ ${ }^{\text {a }}$ School of Automation \& Electronics Engineering, Qingdao University of Science \& Technology, Qingdao 266042, PRC \\ (phone: +86-532-8402-2684; fax: +86-532-8402-2687; e-mail: ronghu_chi@hotmail.com) \\ ${ }^{\mathrm{b}}$ School of Mathematics and Physics, Qingdao University of Science and Technology, Qingdao 266042, PRC (e-mail: rkzhang@qust.edu.cn) \\ ${ }^{\mathrm{c}}$ Advanced Control Systems Lab, School of Electronics \& Information Engineering, Beijing Jiaotong University, Beijing 100044, PRC \\ (e-mail: zhshhou@bjtu.edu.cn, shtjin@bjtu.edu.cn) \\ ${ }^{\mathrm{d}}$ Department of Chemical and Materials Engineering, University of Alberta, Edmonton, AB, Canada T6G 2G6 (bhuang@ualberta.ca)
}

\begin{abstract}
A constrained optimal ILC for a class of nonlinear and non-affine systems, without requiring any explicit model information except for the input and output data, is proposed in this work. In order to address the nonlinearities, an iterative dynamic linearization method without omitting any information of the original plant is introduced in the iteration direction. The derived linearized data model is equivalent to the original nonlinear system and reflects the real-time dynamics of the controlled plant, rather than a static approximate model. By transferring all the constraints on the system output, control input, and the change rate of input signals into a linear matrix inequality, a novel constrained data-driven optimal ILC is developed by minimizing a predesigned objective function. The optimal learning gain is unfixed and updated iteratively according to the input and output measurements, which enhances the flexibility regarding modifications and expansions of the controlled plant. The results are further extended to the point-to-point control tasks where the exact tracking performance is required only at certain points and a constrained data-driven optimal point-to-point ILC is proposed by only utilizing the error measurements at the specified points only.
\end{abstract}

Key words: Data-driven control, Iterative learning control, Constrained nonlinear systems, Quadratic programming, Point-to-point tracking tasks 


\section{Introduction}

Iterative learning control (ILC) [1-3] was proposed for repetitive control tasks on a finite time interval. The basic methodology of ILC is to use the prior knowledge of a repetitive process from previous operations to progressively improve tracking performance from iteration to iteration. It has been proved effective for perfect tracking and has achieved a great success in both theory and applications.

In real process industries, a soft limiter is often added directly into the controller to prevent overly large control input signals [4]. However, such a limiter makes the control systems highly nonlinear and may introduce instability. It is also worth pointing out that the input saturation will bring more serious influence on ILC systems because ILC is virtually an integral action along the iteration direction [5]. Recently, several ILC schemes with input saturation have been proposed under the framework of contraction mapping [69]. However, the contraction mapping based ILC may cause some poor transient performance, even divergence at some time instants. Therefore, an adaptive ILC with input saturation has been proposed in [5] by introducing a composite energy function. In [10], a "reference governor" based ILC was proposed for input constraint systems. By using RG, the reference signal can be re-designed such that the tracking objective is realizable. The authors in [11] proposed a unified design framework of ILC to deal with nonlinear input uncertainties including input saturations.

Besides the input saturation, the output constraints should also be of a greater concern from a practical viewpoint. For example, the train operation system is constrained not only by the input force because of its mechanical features, but also by its operation speed because of its overspeed limits. The latter seems more important to ensure operation performance and safety. Generally, the position and/or speed is required to be constrained in most motion systems.

In the expository overview of ILC [12], optimal ILC [13-16] is revisited and classified as a connection between ILC and optimal control. By using a predesigned objective function, various practical issues such as constraints on the system output and control input, disturbances, measurement noises, and model errors 
can be considered in a rigorous and systematic manner. Recently, some model predictive control (MPC) based ILC schemes were also proposed in the literatures [17-21] by incorporating the input and output constraints into the optimization problem. For example, in [21], a two-stage algorithm has been devised by modifying and combining the existing Q-ILC and BMPC techniques. Further, based on a 2D piecewise linear description of a batch process, the authors in [22] proposed a constrained ILC approach by solving a linear matrix inequality for performance optimization. However, the main limitation is the requirement of an exact linear model of the controlled plant to guarantee a satisfactory control performance. In order to obtain a linear model for the nonlinear system, two open-loop tests have to be carried out where the difference between the two inputs is kept constant throughout the batch.

To handle broader system nonlinearities and uncertainties, adaptive ILC [23-26] was proposed by exploiting the use of energy function approaches. Consequently, adaptive ILC with state constraints was discussed in [27] by using barrier composite energy function. An adaptive ILC was presented in [28] for output-constrained systems with both parametric and nonparametric uncertainties by introducing a new barrier composite energy function. But, the model structure of the controlled system must be available as a priori

\section{knowledge.}

In real world applications, however, the model-based control approaches may encounter many challegences with unsatisfactory performance because it is increasingly difficult to gain an exact model of a complex process with increasing scale and complexity. Even if a mathematical model is obtained by firstprinciples or identification techniques, such a system is not linear in general and the unmodeled uncertainties inevitably exist, which would lead to a poor robustness and lower reliability of the control system. Thus, it is desired to develop a control method less dependent on an explicite model, which is the motivation of data-driven control: the controllers design uses only the input and output measurement data of a plant and the controller itself does not contain any explicite model information of the plant [29-32].

Recently, a data-driven constrained norm-optimal iterative learning control framework for linear timeinvariant systems is proposed in [33], where the system's impulse response is estimated using input and 
output measurements from previous iterations. However, the control performance mainly depends on the estimation precision of the impulse response. To guarantee the estimate values to be equal to the true ones exactly, it is required that the control plant is LTI and no measurement noise or other disturbances are present. More recently, a data-driven terminal ILC [34] is proposed for a special repetitive industrial process where the system input is constant at all sampling instants in a same batch and all the output measurements are not measurable except for the terminal output only. As a consequence, a unified data-driven optimal design framework for generalized iterative learning control [35] is proposed for three control tasks, namely complete trajectory tracking, multiple intermediate points tracking, and single terminal point tracking, respectively. However, the problems of system constraints have not been addressed in the literatures [34, 35].

Motivated by the above discussion, in this work we consider the input and output constraints and propose a constrained data-driven optimal iterative learning control (constrained-DDOILC) for a class of nonlinear systems directly. Before proceeding to the controller design, an iterative dynamical linearization method is revisited with supervector formulation such that the nonlinear plant can be transferred into a linear output function with respect to control inputs and the initial states. This linearization is completely equivalent to the original nonlinear process without neglecting any higher order term and in theory the problem of unmodeled uncertainties do not exist consequently. The parameters in the obtained iterative dynamic linear data model do not have special physical meaning and can be updated iteratively according to the input and output measurements only.

Similar to the Q-ILC schemes, an objective function of control input is designed with a penalty term on the iterative input change. The constraints on the bound of control input and the bound of system output, as well as the change rate of the control input across iterations, are transferred into a linear matrix inequality. Then, the constrained-DDOILC law is obtained by minimizing the designed objective function under this LMI condition. Different from the Q-ILC, the proposed constrained-DDOILC is a data-driven control approach, where no explicit mathematical model, either obtained by first-principles or by identification techniques, is needed. The optimal learning gain of the constrained-DDOILC can be iteratively estimated using 
the real-time input and output data obtained from the system operation, rather than being fixed as that in the Q-ILCs, which makes the proposed constrained-DDOILC more flexible for the modifications or expansions of the controlled plant.

In many practical applications, the objective is to repeatedly track a motion profile in which the tracking errors are of concern only at certain points, instead of every point along the trajectory. For such a control scenario, if removing the unnecessary constraints on the free points other than the specified necessary ones that the plant has to follow, some additional control performance, such as the reduced computation effort and faster convergent speed, could be expected [35]. Several point-to-point ILC (PTP-ILC) approaches have been proposed by using the error information at the given points only [35-38], where, however, the problems of input and output constraints are not well discussed except for [38]. Moreover, most of them are model-based [36-38].

Therefore, this work also extends the proposed constrained-DDOILC to point-to-point motion systems for tracking multiple intermediate points. And a constrained data-driven optimal point-to-point ILC (constrained-DDOPTPILC) is proposed by only utilizing the error measurements at the specified points. Rigorous analysis shows that both of the proposed approaches can achieve a monotonic convergence along the iteration axis. Simulation results are further provided to verify the effectiveness of the proposed constrained-DDOILC and Constrained-DDOPTPILC.

This paper significantly extends the previous work [35]. It improves the design of the DDOILC and DDOPTPILC algorithms by considering the constraints commonly existed in the industrial processes such that the proposed method is always applicable to constrained input and output. The comparative results in the following simulation section also show the significance of this extension since a better control performance can be obtained by the proposed constrained methods. This work is also a significant improvement of traditional Q-ILC for widely existed nonlinear applications, where the control performance is free from the assumption of the linear model, and no linear approximation model is required when applying the proposed constrained methods into nonlinear plants. 
The rest of this paper is organized as follows. Section 2 reviews the iterative dynamical linearization and the transfer of the constraints. Section 3 proposes a constrained-DDOILC to track a desired finite trajectory with detail convergence analysis. Section 4 extends the results to the problem of tracking some specified multiple intermediate pass points rather than all the points. Three simulations are given in Section 5 to verify the effectiveness of the proposed approaches. Conclusions are given in Section 6.

\section{Iterative Dynamical Linearization and Linear Matrix Inequality of Constraints}

\subsection{Iterative Dynamical Linearization}

Consider a nonlinear SISO discrete-time system repetitively operating over a finite time interval as follows,

$$
y_{k}(t+1)=f\left(y_{k}(t), \cdots, y_{k}\left(t-n_{y}\right), u_{k}(t), \cdots, u_{k}\left(t-n_{u}\right)\right)
$$

where $y_{k}(t)$ and $u_{k}(t)$ are the system output and input; $f(\cdot)$ is an unknown nonlinear function and continuously differentiable; $n_{y}$ and $n_{u}$ are two unknown positive integers denoting the orders of system output and input; assume $n_{u}<n_{y}$ in this work; $t \in\{0, \cdots, N\}$, and $N$ is an integer denoting the endpoint of the finite time interval; $k \in\{0,1, \cdots, \infty\}$ denotes the iteration number.

In the following discussion, it is supposed that $u_{k}(t)=0$ and $y_{k}(t)=0$ for all $t<0$. And two other assumptions are also made on the system (1), shown as follows.

Assumption 1: The system initial state $y_{k}(0)$ is unchanged for all iterations, that is, $y_{k}(0)=c, \forall k \in Z^{+}$, where $c$ is a constant.

Assumption 2: Nonlinear function $f(\cdot)$ is globally Lipschitz, that is,

$$
\left|f\left(\boldsymbol{x}_{1}, \boldsymbol{u}_{1}\right)-\boldsymbol{g}\left(\boldsymbol{x}_{2}, \boldsymbol{u}_{2}\right)\right| \leq \bar{L}_{x}\left\|\boldsymbol{x}_{1}-\boldsymbol{x}_{2}\right\|+\bar{L}_{u}\left\|\boldsymbol{u}_{1}-\boldsymbol{u}_{2}\right\|
$$

where $\bar{L}_{x}<\infty$ and $\bar{L}_{u}<\infty$ are two positive Lipschitz constants. 
Remark 1. Assumption 1 is a common condition in the ILC analysis. It means that the controlled plant must start from the same position for every iterative run.

Similar to [35], one can gain a new expression of the system output series formulated by the initial states and system input, shown in equations (2) - (6),

$$
\begin{aligned}
& y_{k}(1)=f\left(y_{k}(0), u_{k}(0)\right)=g^{0}\left(y_{k}(0), u_{k}(0)\right) \\
& y_{k}(2)=f\left(y_{k}(1), y_{k}(0), u_{k}(1), u_{k}(0)\right)=f\left(g^{0}\left(y_{k}(0), u_{k}(0)\right), y_{k}(0), u_{k}(1), u_{k}(0)\right) \\
& =g^{1}\left(y_{k}(0), u_{k}(0), u_{k}(1)\right) \\
& y_{k}\left(n_{u}+1\right)=f\left(y_{k}\left(n_{u}\right), \cdots, y_{k}\left(n_{u}-n_{y}\right), u_{k}\left(n_{u}\right), u_{k}\left(n_{u}-1\right), \cdots, u_{k}(0)\right) \\
& =f\left(g^{n_{u}-1}\left(y_{k}(0), u_{k}(0), \cdots, u_{k}\left(n_{u}-1\right)\right), g^{n_{u}-2}\left(y_{k}(0), u_{k}(0), \cdots, u_{k}\left(n_{u}-2\right)\right)\right. \\
& \left., \cdots, g^{n_{u}-n_{y}-1}\left(y_{k}(0), u_{k}(0), \cdots, u_{k}\left(n_{u}-n_{y}-1\right)\right), u_{k}\left(n_{u}\right), u_{k}\left(n_{u}-1\right), \cdots, u_{k}(0)\right) \\
& =g^{n_{u}}\left(y_{k}(0), u_{k}(0), \cdots, u_{k}\left(n_{u}\right)\right) \\
& y_{k}\left(n_{y}+1\right)=f\left(y_{k}\left(n_{y}\right), \cdots, y_{k}(0), u_{k}\left(n_{y}\right), u_{k}\left(n_{y}-1\right), \cdots, u_{k}(0)\right) \\
& =f\left(g^{n_{y}-1}\left(y_{k}(0), u_{k}(0), \cdots, u_{k}\left(n_{y}-1\right)\right), g^{n_{y}-2}\left(y_{k}(0), u_{k}(0), \cdots, u_{k}\left(n_{y}-2\right)\right)\right. \\
& \left., \cdots, g^{0}\left(y_{k}(0), u_{k}(0)\right), y_{k}(0), u_{k}\left(n_{y}\right), u_{k}\left(n_{y}-1\right), \cdots, u_{k}(0)\right) \\
& =g^{n_{y}}\left(y_{k}(0), u_{k}(0), \cdots, u_{k}\left(n_{y}\right)\right) \\
& y_{k}(N)=f\left(y_{k}(N-1), \cdots, y_{k}\left(N-n_{y}-1\right), u_{k}(N-1), u_{k}(N-2), \cdots, u_{k}\left(N-n_{u}-1\right)\right) \\
& =f\left(g^{N-2}\left(y_{k}(0), u_{k}(0), \cdots, u_{k}(N-2)\right), g^{N-3}\left(y_{k}(0), u_{k}(0), \cdots, u_{k}(N-3)\right)\right. \\
& \left., \cdots, g^{N-n_{y}-2}\left(y_{k}(0), u_{k}(0), \cdots, u_{k}\left(N-n_{y}-2\right)\right), u_{k}(N-1), u_{k}(N-2), \cdots, u_{k}\left(N-n_{u}-1\right)\right) \\
& =g^{N-1}\left(y_{k}(0), u_{k}(0), \cdots, u_{k}(N-1)\right)
\end{aligned}
$$

where $g^{i}(\cdot), i=0, \cdots, N-1$, is a compound function of $f(\cdot)$.

Remark 2. In this work, we only need to know the existence of $g^{i}(\cdot), i=0, \cdots, N-1$, without requiring its exact expression because nonlinear function $f(\cdot)$ is unknown. As long as $f(\cdot)$ exists, $g^{i}(\cdot)$, $i=0, \cdots, N-1$, must exist too as a corresponding compound function. Further, according to the principle in Advanced Mathematics, one can infer that $g^{i}(\cdot)$ has the same properties as that of the nonlinear function $f(\cdot)$, such as continuity, differentiability, boundedness, and so on. 
For simplicity, define three vectors $\boldsymbol{U}_{k}=\left[u_{k}(0), u_{k}(1), \cdots, u_{k}(N-1)\right]^{T}, \boldsymbol{Y}_{k}=\left[y_{k}(1), y_{k}(2), \cdots, y_{k}(N)\right]^{T}$, and $\boldsymbol{g}(\cdot)=\left[\begin{array}{llll}g^{0}(\cdot) & g^{1}(\cdot) & \cdots & g^{N-1}(\cdot)\end{array}\right]^{T}$. Then, the above equations (2) - (6) can be rewritten in a vector form as

$$
\boldsymbol{Y}_{k}=\boldsymbol{g}\left(y_{k}(0), \boldsymbol{U}_{k}^{T}\right)
$$

In terms of Remark 2, one can modify Assumption 2 as follows.

Assumption 2': The nonlinear function $\boldsymbol{g}(\cdot)$ is globally Lipschitz, that is,

$$
\left\|\boldsymbol{g}\left(x_{1}, \boldsymbol{u}_{1}\right)-\boldsymbol{g}\left(x_{2}, \boldsymbol{u}_{2}\right)\right\| \leq L_{x}\left|x_{1}-x_{2}\right|+L_{u}\left\|\boldsymbol{u}_{1}-\boldsymbol{u}_{2}\right\|
$$

where $L_{x}<\infty$ and $L_{u}<\infty$ are two positive Lipschitz constants.

According to [35], the iterative dynamical linearization of nonlinear system (1) can be finally given as follows,

$$
\Delta \boldsymbol{Y}_{k}=\frac{\partial \boldsymbol{g}_{\text {opt }}}{\partial \boldsymbol{U}_{k}^{T}}\left[\boldsymbol{U}_{k}-\boldsymbol{U}_{k-1}\right]=\boldsymbol{\Phi}_{k} \Delta \boldsymbol{U}_{k}
$$

where $\boldsymbol{\Phi}_{k}=\frac{\partial \boldsymbol{g}_{\text {opt }}}{\partial \boldsymbol{U}_{k}^{T}}=\left[\begin{array}{cccc}\frac{\partial g_{o p t}^{0}}{\partial u_{k}(0)} & 0 & \cdots & 0 \\ \frac{\partial g_{o p t}^{1}}{\partial u_{k}(0)} & \frac{\partial g_{o p t}^{1}}{\partial u_{k}(1)} & \cdots & 0 \\ \vdots & \vdots & \ddots & \vdots \\ \frac{\partial g_{o p t}^{N-1}}{\partial u_{k}(0)} & \frac{\partial g_{o p t}^{N-1}}{\partial u_{k}(1)} & \cdots & \frac{\partial g_{o p t}^{N-1}}{\partial u_{k}(N-1)}\end{array}\right] \in R^{N \times N}$, denoting the optimal partial derivative

values of nonlinear function $\boldsymbol{g}$ with respect to $\boldsymbol{U}_{k}$ at some point in the interval $\left[\boldsymbol{U}_{k}, \boldsymbol{U}_{k-1}\right] ; \Delta \boldsymbol{Y}_{k}=\boldsymbol{Y}_{k}-\boldsymbol{Y}_{k-1}$ and $\Delta \boldsymbol{U}_{k}=\boldsymbol{U}_{k}-\boldsymbol{U}_{k-1}$. Moreover, $\sup _{k}\left\|\boldsymbol{\Phi}_{k}\right\| \leq L_{u}$ due to Assumption 2'.

\subsection{Linear matrix inequality of the constraints}

In this work, three kinds of constraints are taken into consideration, shown as follows.

(1) Constraints on the bounds of input values,

$$
\boldsymbol{U}^{\text {low }} \leq \boldsymbol{U}_{k+1} \leq \boldsymbol{U}^{h i}
$$


(2) Constraints on the iterative change rate of the control input along with iteration axis,

$$
\Delta \boldsymbol{U}^{\text {low }} \leq \Delta \boldsymbol{U}_{k+1} \leq \Delta \boldsymbol{U}^{h i}
$$

(3) Constraints on the bounds of system outputs

$$
\boldsymbol{Y}^{\text {low }} \leq \boldsymbol{Y}_{k+1} \leq \boldsymbol{Y}^{h i}
$$

where $\boldsymbol{U}^{\text {low }}, \boldsymbol{U}^{h i}, \Delta \boldsymbol{U}^{\text {low }}, \Delta \boldsymbol{U}^{h i}, \boldsymbol{Y}^{\text {low }}$, and $\boldsymbol{Y}^{\text {hi }}$ are some constant vectors, which are known exactly, $\Delta \boldsymbol{U}_{k+1}=\boldsymbol{U}_{k+1}-\boldsymbol{U}_{k}$.

Subtracting $\boldsymbol{U}_{k}$ from both sides of the inequality (9), one can obtain

$$
\boldsymbol{U}^{\text {low }}-\boldsymbol{U}_{k} \leq \Delta \boldsymbol{U}_{k+1} \leq \boldsymbol{U}^{h i}-\boldsymbol{U}_{k}
$$

Subtracting $\boldsymbol{Y}_{k}$ from both sides of (11), yields,

$$
\boldsymbol{Y}^{\text {low }}-\boldsymbol{Y}_{k} \leq \Delta \boldsymbol{Y}_{k+1} \leq \boldsymbol{Y}^{h i}-\boldsymbol{Y}_{k}
$$

According to (8), the following inequalities are satisfied,

$$
\boldsymbol{Y}^{\text {low }}-\boldsymbol{Y}_{k} \leq \boldsymbol{\Phi}_{k+1} \Delta \boldsymbol{U}_{k+1} \leq \boldsymbol{Y}^{h i}-\boldsymbol{Y}_{k}
$$

Combing (10), (12) and (14), the above constraints can be transferred to a linear matrix inequality as follows,

$$
\boldsymbol{A}_{k}^{\prime} \Delta \boldsymbol{U}_{k+1} \leq \boldsymbol{b}_{k}^{\prime}
$$

where $\boldsymbol{A}_{k}^{\prime}=\left[\begin{array}{c}\boldsymbol{I} \\ -\boldsymbol{I} \\ \boldsymbol{I} \\ -\boldsymbol{I} \\ \boldsymbol{\Phi}_{k+1} \\ -\boldsymbol{\Phi}_{k+1}\end{array}\right] \quad \boldsymbol{b}_{k}^{\prime}=\left[\begin{array}{c}\boldsymbol{U}^{h i}-\boldsymbol{U}_{k} \\ -\boldsymbol{U}^{\text {low }}+\boldsymbol{U}_{k} \\ \Delta \boldsymbol{U}^{h i} \\ -\Delta \boldsymbol{U}^{\text {low }} \\ \boldsymbol{Y}^{h i}-\boldsymbol{Y}_{k} \\ -\boldsymbol{Y}^{\text {low }}+\boldsymbol{Y}_{k}\end{array}\right]$

Let $\Delta \boldsymbol{U}_{k}^{l o w^{*}}=\max \left(\boldsymbol{U}^{\text {low }}-\boldsymbol{U}_{k}, \Delta \boldsymbol{U}^{\text {low }}\right)$, and $\Delta \boldsymbol{U}_{k}^{h i^{*}}=\min \left(\boldsymbol{U}^{h i}-\boldsymbol{U}_{k}, \Delta \boldsymbol{U}^{h i}\right)$; then the linear matrix inequality (15) becomes,

$$
\boldsymbol{A}_{k} \Delta \boldsymbol{U}_{k+1} \leq \boldsymbol{b}_{k}
$$


where $\boldsymbol{A}_{k}=\left[\begin{array}{c}\boldsymbol{I} \\ -\boldsymbol{I} \\ \boldsymbol{\Phi}_{k+1} \\ -\boldsymbol{\Phi}_{k+1}\end{array}\right]$ and $\boldsymbol{b}_{k}=\left[\begin{array}{c}\Delta \boldsymbol{U}_{k}^{h i^{*}} \\ -\Delta \boldsymbol{U}_{k}^{l o w^{*}} \\ \boldsymbol{Y}^{h i}-\boldsymbol{Y}_{k} \\ -\boldsymbol{Y}^{l o w}+\boldsymbol{Y}_{k}\end{array}\right]$.

Given a desired trajectory $y_{d}(t), t \in\{0, \cdots, N\}$. The objective is to find an optimal control input $u_{k}(t)$ under the above three kinds of constraints (9) - (11) such that the system output $y_{k}(t)$ can track $y_{d}(t)$ exactly for all time instants. That is, the tracking error $\boldsymbol{E}_{k}=\boldsymbol{Y}_{d}-\boldsymbol{Y}_{k}$ converges to zero as the iteration number $k$ approaches to infinity where $\boldsymbol{Y}_{d}=\left[y_{d}(1), y_{d}(2), \cdots, y_{d}(N)\right]^{T} \in R^{N}$.

\section{Constrained Data-driven Optimal Iterative Learning Control}

\subsection{Controller Design}

The constraint optimal ILC law is proposed by minimizing the following quadratic performance index function with respect to $U_{k+1}$ under the linear matrix inequality of the constraints (16),

$$
J\left(\boldsymbol{E}_{k+1}, \boldsymbol{U}_{k+1}\right)=\frac{1}{2}\left\{\boldsymbol{E}_{k+1}^{T} \boldsymbol{Q} \boldsymbol{E}_{k+1}+\Delta \boldsymbol{U}_{k+1}^{T} \boldsymbol{R} \Delta \boldsymbol{U}_{k+1}\right\}
$$

where $\boldsymbol{Q}$ and $\boldsymbol{R}$ are positive definite weighting matrices.

According to (8), it is easy to get

$$
\boldsymbol{E}_{k+1}=\boldsymbol{Y}_{d}-\boldsymbol{Y}_{k+1}=\boldsymbol{Y}_{d}-\boldsymbol{Y}_{k}-\Delta \boldsymbol{Y}_{k+1}=\boldsymbol{E}_{k}-\boldsymbol{\Phi}_{k+1} \Delta \boldsymbol{U}_{k+1}
$$

Substituting (18) into (17), yields

$$
J\left(\boldsymbol{E}_{k+1}, \boldsymbol{U}_{k+1}\right)=\frac{1}{2}\left\{\Delta \boldsymbol{U}_{k+1}^{T}\left(\boldsymbol{\Phi}_{k+1}^{T} \boldsymbol{Q} \boldsymbol{\Phi}_{k+1}+\boldsymbol{R}\right) \Delta \boldsymbol{U}_{k+1}-2 \boldsymbol{E}_{k}^{T} \boldsymbol{Q} \boldsymbol{\Phi}_{k+1} \Delta \boldsymbol{U}_{k+1}+\boldsymbol{E}_{k}^{T} \boldsymbol{Q} \boldsymbol{E}_{k}\right\}
$$

Then the optimal ILC law can be obtained by minimizing (19) with the constraints (16), that is, 


$$
\left\{\begin{array}{l}
\min _{\boldsymbol{U}_{k+1}} J\left(\boldsymbol{E}_{k+1}, \boldsymbol{U}_{k+1}\right)=\min _{\boldsymbol{U}_{k+1}} \frac{1}{2}\left\{\Delta \boldsymbol{U}_{k+1}^{T}\left(\boldsymbol{\Phi}_{k+1}^{T} \boldsymbol{Q} \boldsymbol{\Phi}_{k+1}+\boldsymbol{R}\right) \Delta \boldsymbol{U}_{k+1}-2 \boldsymbol{E}_{k}^{T} \boldsymbol{Q} \boldsymbol{\Phi}_{k+1} \Delta \boldsymbol{U}_{k+1}+\boldsymbol{E}_{k}^{T} \boldsymbol{Q} \boldsymbol{E}_{k}\right\} \\
\text { s.t. } \quad \boldsymbol{A}_{k} \Delta \boldsymbol{U}_{k+1} \leq \boldsymbol{b}_{k}
\end{array}\right.
$$

where $\boldsymbol{\Phi}_{k+1}$ is unknown. Therefore, an iterative updating law is presented in this work to estimate $\boldsymbol{\Phi}_{k+1}$ by using the input and output data only. Since $\boldsymbol{\Phi}_{k+1}$ is a lower triangular matrix and all the upper triangular elements are zeros, so only the nonzero elements of $\boldsymbol{\Phi}_{k+1}$ are estimated to reduce the computational complexity. According to [35], the parameter updating law of $\boldsymbol{\Phi}_{k+1}$ is given as follows,

$$
\hat{\boldsymbol{\varphi}}_{k+1}^{t}(t)=\hat{\boldsymbol{\varphi}}_{k}^{t}(t)+\frac{\eta\left(\Delta y_{k}(t+1)-\hat{\boldsymbol{\varphi}}_{k}^{t}(t) \Delta \boldsymbol{u}_{k}(t)\right) \Delta \boldsymbol{u}_{k}^{T}(t)}{\mu+\left\|\Delta \boldsymbol{u}_{k}(t)\right\|^{2}}
$$

where $\hat{\boldsymbol{\varphi}}_{k+1}^{t}(t)$ is the estimated value of $\boldsymbol{\varphi}_{k+1}^{t}(t)$, and $\boldsymbol{\varphi}_{k+1}^{t}(t)$ denotes the nonzero vector of the (t+1)-th row of $\boldsymbol{\Phi}_{k+1}$. For example, $\boldsymbol{\varphi}_{k+1}^{0}(0)=\frac{\partial g^{0}}{\partial u_{k+1}(0)}$ consists of the nonzero element of the first row of $\boldsymbol{\Phi}_{k+1}$, $\boldsymbol{\varphi}_{k+1}^{1}(1)=\left[\frac{\partial g^{1}}{\partial u_{k+1}(0)}, \frac{\partial g^{1}}{\partial u_{k+1}(1)}\right]$ consists of the nonzero elements of the second row of $\boldsymbol{\Phi}_{k+1}$, and $\boldsymbol{\varphi}_{k+1}^{t}(t)=\left[\frac{\partial g^{t}}{\partial u_{k+1}(0)}, \frac{\partial g^{t}}{\partial u_{k+1}(1)}, \cdots, \frac{\partial g^{t}}{\partial u_{k+1}(t)}\right]$ consists of the nonzero elements of the $(t+1)$-th row of $\boldsymbol{\Phi}_{k+1}$. Correspondingly, $\boldsymbol{u}_{k}(t)=\left[u_{k}(0), u_{k}(1), \cdots, u_{k}(t)\right]^{T} \in R^{t+1}$ is a varying dimension vector. That is, if $t=0$, then $\boldsymbol{u}_{k}(0)=\left[u_{k}(0)\right] ; \quad$ if $t=1$, then $\boldsymbol{u}_{k}(1)=\left[u_{k}(0), u_{k}(1)\right]^{T} . \mu>0$ is a weighting factor and and $0<\eta<2$ is a step-size factor.

Finally, the proposed constrained optimal ILC law can be obtained by solving the following quadratic programming $(\mathrm{QP})$ problem according to the updating law (21),

$$
\left\{\begin{array}{l}
\min _{\boldsymbol{U}_{k+1}} J\left(\boldsymbol{E}_{k+1}, \boldsymbol{U}_{k+1}\right)=\min _{\boldsymbol{U}_{k+1}} \frac{1}{2}\left\{\Delta \boldsymbol{U}_{k+1}^{T}\left(\hat{\boldsymbol{\Phi}}_{k+1}^{T} \boldsymbol{Q} \hat{\boldsymbol{\Phi}}_{k+1}+\boldsymbol{R}\right) \Delta \boldsymbol{U}_{k+1}-2 \boldsymbol{E}_{k}^{T} \boldsymbol{Q} \hat{\boldsymbol{\Phi}}_{k+1} \Delta \boldsymbol{U}_{k+1}+\boldsymbol{E}_{k}^{T} \boldsymbol{Q} \boldsymbol{E}_{k}\right\} \\
\text { s.t. } \quad \hat{\boldsymbol{A}}_{k} \Delta \boldsymbol{U}_{k+1} \leq \boldsymbol{b}_{k}
\end{array}\right.
$$


where $\hat{\boldsymbol{A}}_{k}=\left[\begin{array}{c}\boldsymbol{I} \\ -\boldsymbol{I} \\ \hat{\boldsymbol{\Phi}}_{k+1} \\ -\hat{\boldsymbol{\Phi}}_{k+1}\end{array}\right]$.

That is, the proposed constraint data-driven optimal ILC is constructed by (21) and (22).

Remark 3. From (21) and (22), it can be seen that no system model information except for the I/O data is used in the algorithms and thus the proposed approach is data-driven. Further, the learning gain of (22) can be iteratively updated by using (21), so the proposed approach can be more robust to the system uncertainties.

\subsection{Convergence analysis}

Before proceeding to presenting the convergence theorem, other two assumptions are given for a rigor of analysis.

Assumption 3: For the reference trajectory $\boldsymbol{Y}_{d}$, there exists control input $\boldsymbol{U}_{\infty}$ such that the output of system (1) can track $\boldsymbol{Y}_{d}$ exactly. That is, $\exists \boldsymbol{U}_{\infty}$ such that $\boldsymbol{Y}_{d}-\boldsymbol{\Phi}_{k} \boldsymbol{U}_{\infty}=\boldsymbol{0}, \quad \boldsymbol{U}^{\text {low }} \leq \boldsymbol{U}_{\infty} \leq \boldsymbol{U}^{h i}$ and $\boldsymbol{Y}^{\text {low }} \leq \boldsymbol{Y}_{d} \leq \boldsymbol{Y}^{h i}$. Moreover, $\Delta \boldsymbol{U}^{\text {low }}<0$ and $\Delta \boldsymbol{U}^{h i}>0$.

Assumption 4: Output constraints are satisfied when $\boldsymbol{E}_{k}=\boldsymbol{0}$.

Remark 4. The assumptions 3 and 4 are similar to that in [16]. They are clearly necessary in order for any algorithm to achieve zero tracking error while satisfying the constraints. Assumption 3 is similar to the reachability condition of a control system. If assumption 3 does not hold, it implies that the control task is not achievable and the tracking error can only converge to a bounded range.

Theorem 1: If nonlinear system (1) with input and output constraints (9) - (11) satisfies assumptions 1 4, then applying the proposed constrained-DDOILC approach (21) - (22), one can guarantee that: a) The 
estimation of $\hat{\boldsymbol{\Phi}}_{k}$ is bounded for all time instants and iterations; b) The change rate of input along the iteration direction, $\Delta \boldsymbol{U}_{k}$, converges to zero, i.e., $\lim _{k \rightarrow \infty} \Delta \boldsymbol{U}_{k}=\boldsymbol{0}$; (c) the tracking error $\boldsymbol{E}_{k}$ also converges to zero iteratively, i.e., $\lim _{k \rightarrow \infty} \boldsymbol{E}_{k}=\boldsymbol{0}$; and (d) both the system input and output are bounded for all time instants and iterations.

Proof. The boundedness of $\hat{\boldsymbol{\Phi}}_{k}$ has been proved in [35]. According to the performance index function, it is clear that

$$
J\left(\boldsymbol{E}_{k+1}, \boldsymbol{U}_{k+1}\right)=\frac{1}{2}\left\{\boldsymbol{E}_{k+1}^{T} \boldsymbol{Q} \boldsymbol{E}_{k+1}+\Delta \boldsymbol{U}_{k+1}^{T} \boldsymbol{R} \Delta \boldsymbol{U}_{k+1}\right\} \geq 0,
$$

where $\left(\boldsymbol{E}_{k+1}, \boldsymbol{U}_{k+1}\right) \in \boldsymbol{\Omega}_{k+1}$ is the optimal solution of $J\left(\boldsymbol{E}_{k+1}, \boldsymbol{U}_{k+1}\right)$ and $\boldsymbol{\Omega}_{k+1}$ is the feasible region defined by system constraints (16).

According to (18), if we fix $\Delta \boldsymbol{U}_{k+1}=\boldsymbol{0}$, one obtains that

$$
\boldsymbol{E}_{k+1}=\boldsymbol{E}_{k}-\boldsymbol{\Phi}_{k+1} \Delta \boldsymbol{U}_{k+1}=\boldsymbol{E}_{k}
$$

Therefore, $\left(\boldsymbol{E}_{k}, \boldsymbol{0}\right) \in \boldsymbol{\Omega}_{k+1}$ is also a feasible point. According to the optimality of the index function (17) at the point $\left(\boldsymbol{E}_{k+1}, \boldsymbol{U}_{k+1}\right)$, the following inequality is established,

$$
J\left(\boldsymbol{E}_{k+1}, \boldsymbol{U}_{k+1}\right) \leq J\left(\boldsymbol{E}_{k}, \boldsymbol{0}\right)=\frac{1}{2}\left\{\boldsymbol{E}_{k}^{T} \boldsymbol{Q} \boldsymbol{E}_{k}\right\}=J\left(\boldsymbol{E}_{k}, \boldsymbol{U}_{k}\right)-\frac{1}{2} \Delta \boldsymbol{U}_{k}^{T} \boldsymbol{R} \Delta \boldsymbol{U}_{k}
$$

which implies that

$$
0 \leq J\left(\boldsymbol{E}_{k+1}, \boldsymbol{U}_{k+1}\right) \leq J\left(\boldsymbol{E}_{0}, \boldsymbol{U}_{0}\right)-\frac{1}{2} \sum_{i=1}^{k} \Delta \boldsymbol{U}_{i}^{T} \boldsymbol{R} \Delta \boldsymbol{U}_{i}
$$

Eq. (26) can be rewritten as, 


$$
0 \leq J\left(\boldsymbol{E}_{k+1}, \boldsymbol{U}_{k+1}\right)+\frac{1}{2} \sum_{i=1}^{k} \Delta \boldsymbol{U}_{i}^{T} \boldsymbol{R} \Delta \boldsymbol{U}_{i} \leq J\left(\boldsymbol{E}_{0}, \boldsymbol{U}_{0}\right) \leq \infty
$$

It is clear from (27) that $\lim _{k \rightarrow \infty} \Delta \boldsymbol{U}_{k}=0$. Conclusion (b) is proved.

Now, we proceed to show that $\left(\boldsymbol{0}, \boldsymbol{U}_{\infty}^{k}\right)$ is also a feasible point, where $\boldsymbol{U}_{\infty}^{k}=\boldsymbol{U}_{\infty}-\boldsymbol{U}_{k}$.

From assumption (A3) we know that

$$
\boldsymbol{U}^{\text {low }} \leq \boldsymbol{U}_{\infty} \leq \boldsymbol{U}^{h i}
$$

It is apparent that

$$
\boldsymbol{U}^{\text {low }}-\boldsymbol{U}_{k} \leq \boldsymbol{U}_{\infty}-\boldsymbol{U}_{k} \leq \boldsymbol{U}^{h i}-\boldsymbol{U}_{k}
$$

From conclusion (b) of Theorem 1, $\Delta \boldsymbol{U}_{k} \rightarrow 0$. According to assumption $3, \Delta \mathbf{U}^{\text {low }}<0$ and $\Delta \mathbf{U}^{h i}>0$, so we have $\Delta \boldsymbol{U}^{\text {low }} \leq \Delta \boldsymbol{U}_{k+1} \leq \Delta \boldsymbol{U}^{h i}$ for sufficiently large $k$. Therefore, the inequality $\Delta \boldsymbol{U}^{\text {low }} \leq \boldsymbol{U}_{\infty}^{k} \leq \Delta \boldsymbol{U}^{h i}$ can be established for large enough $k$.

According to assumption 3, it follows that

$$
\boldsymbol{Y}^{\text {low }}-\boldsymbol{Y}_{k} \leq \boldsymbol{Y}_{d}-\boldsymbol{Y}_{k} \leq \boldsymbol{Y}^{h i}-\boldsymbol{Y}_{k}
$$

and

$$
\boldsymbol{Y}^{\text {low }}-\boldsymbol{Y}_{k} \leq \boldsymbol{\Phi}_{k}\left(\boldsymbol{U}_{\infty}-\boldsymbol{U}_{k}\right) \leq \boldsymbol{Y}^{h i}-\boldsymbol{Y}_{k}
$$

Thus, $\left(\boldsymbol{0}, \boldsymbol{U}_{\infty}^{k}\right)$ is also a feasible point.

Moreover, the optimal solution $\left(\boldsymbol{E}_{k+1}, \boldsymbol{U}_{k+1}\right)$ is a feasible point obviously. By virtue of the convexity of the feasible region $\boldsymbol{\Omega}_{k+1}$, any point between the two feasible points is also feasible. 
Define the vector

$$
\boldsymbol{l}=\left(\boldsymbol{0}, \boldsymbol{U}_{\infty}^{k}\right)^{T}-\left(\boldsymbol{E}_{k+1}, \Delta \boldsymbol{U}_{k+1}\right)^{T}=\left(-\boldsymbol{E}_{k+1}, \boldsymbol{U}_{\infty}^{k}-\Delta \boldsymbol{U}_{k+1}\right)^{T}
$$

The directional derivative of $J\left(\boldsymbol{E}_{k+1}, \boldsymbol{U}_{k+1}\right)$ along the direction of the partial derivatives $\boldsymbol{l}$ can be obtained as

$$
\begin{aligned}
\left.\Delta J^{T}\left(\boldsymbol{E}_{k+1}, \boldsymbol{U}_{k+1}\right)\right|_{\left(\boldsymbol{E}_{k+1}, \Delta \boldsymbol{U}_{k+1}\right)} \cdot \boldsymbol{l} & =\left[\boldsymbol{E}_{k+1}^{T}, \Delta \boldsymbol{U}_{k+1}^{T}\right] \cdot\left[\begin{array}{c}
-\boldsymbol{E}_{k+1} \\
\boldsymbol{U}_{\infty}^{k}-\Delta \boldsymbol{U}_{k+1}
\end{array}\right] \\
& =-\boldsymbol{E}_{k+1}^{T} \boldsymbol{E}_{k+1}+\Delta \boldsymbol{U}_{k+1}^{T} \boldsymbol{U}_{\infty}^{k}-\Delta \boldsymbol{U}_{k+1}^{T} \Delta \boldsymbol{U}_{k+1}
\end{aligned}
$$

Since $\left(\boldsymbol{E}_{k+1}, \Delta \boldsymbol{U}_{k+1}\right)$ is the optimal point to minimize the index function $J\left(\boldsymbol{E}_{k+1}, \boldsymbol{U}_{k+1}\right)$, clearly the cost of function $J\left(\boldsymbol{E}_{k+1}, \boldsymbol{U}_{k+1}\right)$ at $\left(\boldsymbol{o}, \boldsymbol{U}_{\infty}^{k}\right)$ is no less than that at the point of $\left(\boldsymbol{E}_{k+1}, \Delta \boldsymbol{U}_{k+1}\right)$. Thus, the directional derivative of $J\left(\boldsymbol{E}_{k+1}, \boldsymbol{U}_{k+1}\right)$ along the direction $\boldsymbol{l}$ is no less than zero. From (33), it is derived that

$$
\Delta \boldsymbol{U}_{k+1}^{T} \boldsymbol{U}_{\infty}^{k} \geq \boldsymbol{E}_{k+1}^{T} \boldsymbol{E}_{k+1}+\Delta \boldsymbol{U}_{k+1}^{T} \Delta \boldsymbol{U}_{k+1} \geq 0
$$

It is directly concluded from (34) that $\lim _{k \rightarrow \infty} \boldsymbol{E}_{k}=0$ because $\lim _{k \rightarrow \infty} \Delta \boldsymbol{U}_{k}=0$ has been shown. So we get conclusion (c) of Theorem 1.

\section{Constrained Data-driven Optimal PTP-ILC}

\subsection{Controller Design}

In this section, the control objective is to track only certain desired output points $y_{d}\left(t_{1}\right), y_{d}\left(t_{2}\right), \cdots, y_{d}\left(t_{M}\right)$ at time instants of $\left\{t_{1}, t_{2}, \cdots, t_{M}\right\}, t_{M} \leq N$. Moreover, the system output at points other than the specified ones $\left\{t_{1}, t_{2}, \cdots, t_{M}\right\}$ may be unmeasurable. In this work, therefore, a constrained data-driven optimal point-to-point ILC (constrained-DDOPTPILC) law will be designed by only using the error information obtained from the specified points. 
Define $\overline{\boldsymbol{Y}}_{k}=\left[y_{k}\left(t_{1}\right), y_{k}\left(t_{2}\right), \cdots, y_{k}\left(t_{M}\right)\right]^{T} \in R^{M}$, which is the $t_{i}$-th row of the row vector $\boldsymbol{Y}_{k}, m=1, \cdots, M$. According to [35], we have

$$
\Delta \overline{\boldsymbol{Y}}_{k}=\overline{\boldsymbol{\Phi}}_{k} \Delta \boldsymbol{U}_{k}
$$

where $\overline{\boldsymbol{\Phi}}_{k}=\left[\begin{array}{ccccccccccc}\frac{\partial g_{t_{1}-1}^{*}}{\partial u_{k}(0)} & \frac{\partial g_{t_{1}-1}^{*}}{\partial u_{k}(1)} & \cdots & \frac{\partial g_{t_{1}-1}^{*}}{\partial u_{k}\left(t_{1}-1\right)} & 0 & 0 & \cdots & 0 & 0 & \cdots & 0 \\ \frac{\partial g_{t_{2}-1}^{*}}{\partial u_{k}(0)} & \frac{\partial g_{t_{2}-1}^{*}}{\partial u_{k}(1)} & \cdots & \frac{\partial g_{t_{2}-1}^{*}}{\partial u_{k}\left(t_{1}-1\right)} & \cdots & \frac{\partial g_{t_{2}-1}^{*}}{\partial u_{k}\left(t_{2}-1\right)} & \cdots & 0 & 0 & \cdots & 0 \\ \vdots & \vdots & \ddots & \vdots & & \vdots & \ddots & \vdots & \vdots & \ddots & \vdots \\ \frac{\partial g_{t_{M}-1}^{*}}{\partial u_{k}(0)} & \frac{\partial g_{t_{M}-1}^{*}}{\partial u_{k}(1)} & \cdots & \frac{\partial g_{t_{M}-1}^{*}}{\partial u_{k}\left(t_{1}-1\right)} & \cdots & \frac{\partial g_{t_{M}-1}^{*}}{\partial u_{k}\left(t_{2}-1\right)} & \cdots & \frac{\partial g_{t_{M}-1}^{*}}{\partial u_{k}\left(t_{M}-1\right)} & 0 & \cdots & 0\end{array}\right]^{M \times N}$

Similarly, we also consider three kinds of constraints: bounds on input values, bounds on iterative change rate of the control input, and bounds on system outputs. The first two constraints are the same as that defined in (9) and (10). The constraints on the system output are required only at the specified points $\left\{t_{1}, t_{2}, \cdots, t_{M}\right\}$ for the point-to-point operation systems because the system output at the other points are not necessary. Hence, the constraints on the bounds of system outputs for the point-to-point operation system becomes,

$$
\boldsymbol{Y}^{\text {low }} \leq \overline{\boldsymbol{Y}}_{k+1} \leq \boldsymbol{Y}^{h i}
$$

By following the same steps from (12) to (16), the input and output constraints (9), (10), and (36) are transferred into a linear matrix inequality condition

$$
\overline{\boldsymbol{A}}_{k} \Delta \boldsymbol{U}_{k+1} \leq \overline{\boldsymbol{b}}_{k}
$$

where $\overline{\boldsymbol{A}}_{k}=\left[\begin{array}{c}\boldsymbol{I} \\ -\boldsymbol{I} \\ \overline{\boldsymbol{\Phi}}_{k+1} \\ -\overline{\boldsymbol{\Phi}}_{k+1}\end{array}\right]$ and $\overline{\boldsymbol{b}}_{k}=\left[\begin{array}{c}\Delta \boldsymbol{U}_{k}^{h *^{*}} \\ -\Delta \boldsymbol{U}_{k}^{l o w^{*}} \\ \boldsymbol{Y}^{h i}-\overline{\boldsymbol{Y}}_{k} \\ -\boldsymbol{Y}^{l o w}+\overline{\boldsymbol{Y}}_{k}\end{array}\right]$.

Let $\overline{\boldsymbol{Y}}_{d}=\left[y_{d}\left(t_{1}\right), y_{d}\left(t_{2}\right), \cdots, y_{d}\left(t_{M}\right)\right]^{T} \in R^{M}$ and $\overline{\boldsymbol{E}}_{k}=\overline{\boldsymbol{Y}}_{d}-\overline{\boldsymbol{Y}}_{k}$ denote the tracking errors at the specified points. Define an index function with respect to $\boldsymbol{U}_{k+1}$, 


$$
J\left(\overline{\boldsymbol{E}}_{k+1}, \boldsymbol{U}_{k+1}\right)=\frac{1}{2}\left\{\overline{\boldsymbol{E}}_{k+1}^{T} \boldsymbol{Q} \overline{\boldsymbol{E}}_{k+1}+\Delta \boldsymbol{U}_{k+1}^{T} \boldsymbol{R} \Delta \boldsymbol{U}_{k+1}\right\}
$$

where $\boldsymbol{Q}$ and $\boldsymbol{R}$ are positive definite weighting matrices.

Following the same steps from (18) to (20), the Constrained-DDOPTPILC law is derived by minimizing (38) with the linear matrix inequality constraints (37), that is,

$$
\left\{\begin{array}{l}
\min _{\boldsymbol{U}_{k+1}} J\left(\overline{\boldsymbol{E}}_{k+1}, \boldsymbol{U}_{k+1}\right)=\min _{\boldsymbol{U}_{k+1}} \frac{1}{2}\left\{\Delta \boldsymbol{U}_{k+1}^{T}\left(\overline{\boldsymbol{\Phi}}_{k+1}^{T} \boldsymbol{Q} \overline{\boldsymbol{\Phi}}_{k+1}+\boldsymbol{R}\right) \Delta \boldsymbol{U}_{k+1}-2 \overline{\boldsymbol{E}}_{k}^{T} \boldsymbol{Q} \overline{\boldsymbol{\Phi}}_{k+1} \Delta \boldsymbol{U}_{k+1}+\overline{\boldsymbol{E}}_{k}^{T} \boldsymbol{Q} \overline{\boldsymbol{E}}_{k}\right\} \\
\text { s.t. } \quad \overline{\boldsymbol{A}}_{k} \Delta \boldsymbol{U}_{k+1} \leq \overline{\boldsymbol{b}}_{k}
\end{array}\right.
$$

where $\overline{\boldsymbol{\Phi}}_{k+1}$ is unknown and is estimated iteratively by the following updating law [35],

$$
\hat{\overline{\boldsymbol{\varphi}}}_{k}^{t_{i}-1}\left(t_{i}-1\right)=\hat{\overline{\boldsymbol{\varphi}}}_{k-1}^{t_{i}-1}\left(t_{i}-1\right)+\frac{\eta\left(\Delta y_{k-1}\left(t_{i}\right)-\hat{\overline{\boldsymbol{\varphi}}}_{k-1}^{t_{i}-1}\left(t_{i}-1\right) \Delta \overline{\boldsymbol{u}}_{k-1}\left(t_{i}-1\right)\right) \Delta \overline{\boldsymbol{u}}_{k-1}^{T}\left(t_{i}-1\right)}{\mu+\left\|\Delta \overline{\boldsymbol{u}}_{k-1}\left(t_{i}-1\right)\right\|^{2}}, i=1, \cdots, M,
$$

where $\mu>0$ is a weighting factor and $\eta \in(0,2)$ is a step-size factor; $\hat{\bar{\varphi}}_{k}^{t_{i}-1}\left(t_{i}-1\right)$ is the estimated value of $\overline{\boldsymbol{\varphi}}_{k}^{t_{i}-1}\left(t_{i}-1\right) ; \overline{\boldsymbol{\varphi}}_{k}^{t_{i}-1}\left(t_{i}-1\right)$ denotes the nonzero vector of the $i$-th row of $\overline{\boldsymbol{\Phi}}_{k}$.

Then, the proposed constrained-DDOPTPILC law can be obtained by solving the following quadratic programming (QP) problem according to the updating law (40),

$$
\left\{\begin{array}{l}
\min _{\boldsymbol{U}_{k+1}} J\left(\overline{\boldsymbol{E}}_{k+1}, \boldsymbol{U}_{k+1}\right)=\min _{\boldsymbol{U}_{k+1}} \frac{1}{2}\left\{\Delta \boldsymbol{U}_{k+1}^{T}\left(\hat{\overline{\boldsymbol{\Phi}}}_{k+1}^{T} \boldsymbol{Q} \hat{\overline{\boldsymbol{\Phi}}}_{k+1}+\boldsymbol{R}\right) \Delta \boldsymbol{U}_{k+1}-2 \overline{\boldsymbol{E}}_{k}^{T} \boldsymbol{Q} \hat{\boldsymbol{\Phi}}_{k+1} \Delta \boldsymbol{U}_{k+1}+\overline{\boldsymbol{E}}_{k}^{T} \boldsymbol{Q} \overline{\boldsymbol{E}}_{k}\right\} \\
\text { s.t. } \quad \hat{\overline{\boldsymbol{A}}}_{k} \Delta \boldsymbol{U}_{k+1} \leq \overline{\boldsymbol{b}}_{k}
\end{array}\right.
$$

where $\hat{\overline{\boldsymbol{A}}}_{k}=\left[\begin{array}{c}\boldsymbol{I} \\ -\boldsymbol{I} \\ \hat{\overline{\boldsymbol{\Phi}}}_{k+1} \\ -\hat{\boldsymbol{\Phi}}_{k+1}\end{array}\right]$. 
Remark 5. For the constrained-DDOILC proposed in Section 3, the dimension of $\hat{\boldsymbol{\Phi}}_{k+1}$ in (22) is $N \times N$. So we need to store $3 N+N \times N$ data in the memory and $N \times N$ dimensional matrix calculation is required in the computation. Comparatively, for the multiple intermediate tracking tasks tracking $M$ points only, instead of $N$ points $(M<<N)$, the dimension of $\hat{\boldsymbol{\Phi}}_{k+1}$ in (41) is $M \times M$. And we need to store $3 M+M \times M$ data in the memory, and the dimension of the matrix required for the calculation of the control input is reduced significantly to $M \times M$. It is clear that the constrained-DDOPTPILC proposed in this section has less computational burden and memory size than the constrained-DDOILC.

\subsection{Convergence Analysis}

Two assumptions are given as follows by modifying assumptions 3 and 4 slightly.

Assumption 5: For the desired output points $\overline{\boldsymbol{Y}}_{d}$, there exists control input $\boldsymbol{U}_{\infty}$ such that the output of system (1) can track $\overline{\boldsymbol{Y}}_{d}$ exactly. That is, $\exists \boldsymbol{U}_{\infty}$ such that $\overline{\boldsymbol{Y}}_{d}-\boldsymbol{\Phi}_{k} \boldsymbol{U}_{\infty}=\boldsymbol{0}, \quad \boldsymbol{U}^{\text {low }} \leq \boldsymbol{U}_{\infty} \leq \boldsymbol{U}^{h i}$ and $\boldsymbol{Y}^{\text {low }} \leq \overline{\boldsymbol{Y}}_{d} \leq \boldsymbol{Y}^{h i}$. Moreover, $\Delta \boldsymbol{U}^{\text {low }}<0$ and $\Delta \boldsymbol{U}^{h i}>0$.

Assumption 6: Output constraints are satisfied when $\overline{\boldsymbol{E}}_{k}=\mathbf{0}$.

A similar convergence theorem can be given as follows for the Constrained-DDOPTPILC (40) - (41).

Theorem 2: If the nonlinear system (1) satisfies assumptions 1, 2, 5, and 6, then by applying the proposed constrained-DDOPTPILC (40) - (41), one guarantees that: (a) $\hat{\boldsymbol{\Phi}}_{k}$ is bounded for all time instants and iterations; (b) the convergences of both $\Delta \boldsymbol{U}_{k}$ and $\overline{\boldsymbol{E}}_{k}$ are achieved when the iteration number goes to infinity; and (c) the system outputs at other unprescribed points are bounded for all time instants and iterations.

Proof. The proof of conclusions of Theorem 2 is similar to that of Theorem 1. Now we prove conclusion (c) of Theorem 2. Since the control input is derived by solving the QP problem consisting of (40) and (41), 
it is clear that $\boldsymbol{U}_{k+1}$ is bounded. Then according to (8), $\boldsymbol{Y}_{k}$ is bounded for all iterations because $\sup _{k}\left\|\boldsymbol{\Phi}_{k}\right\| \leq L_{u}$ is bounded by virtue of Assumption 2'.

\section{Simulation Study}

Example 1: Consider a repetitive nonlinear discrete-time system [35],

$$
y_{k}(t+1)=\frac{y_{k}(t)}{1+y_{k}(t)^{3}}+u_{k}(t)^{3}
$$

where $t \in\{0,1, \cdots, 20\}$ is the time instants considered; $k \in\{0,1, \cdots\}$ is the iteration number. The system constraints are set as $-1.0 \leq u_{k}(t) \leq 1.0,-0.25 \leq \Delta u_{k}(t) \leq 0.25$, and $-0.6 \leq y_{k}(t) \leq 0.6$.

The desired trajectory is

$$
y_{d}(t)=0.5 \sin (t \pi / 10)+0.3 \cos (t \pi / 10), t \in\{0,1, \cdots, 20\}
$$

Case 1.1: Tracking the above desired trajectory over the entire time interval $\{0,1, \cdots, 20\}$. That is, a good control performance is required for all finite points of the desired trajectory. Hence, we will use the proposed constrained-DDOILC (21) - (22) for this task.

Select the initial values as $y_{k}(0)=0, u_{0}(t)=0.5$, and $\hat{\boldsymbol{\varphi}}_{0}(t)=0.5$. The controller parameters are set as $\boldsymbol{Q}=\boldsymbol{R}=I, \quad \eta=1, \mu=1$. The simulation results of the Constrained-DDOILC are shown as the red solid lines in figures $1-3$.

Fig. 1 is the output tracking performance of the Constrained-DDOILC, where the black squares are all the discrete points of the desired trajectory and the green solid line is system output profile at the 100-th iteration. Fig. 2 shows the convergence of the maximum absolute tracking errors, defined as $e_{\max }(k)=\max _{t \in\{1,2, \cdots, 20\}}\left\{\left|e_{k}(t)\right|\right\}$, along the iteration direction. Fig. 3 is the corresponding control input profile.

From the simulation results, one can conclude that: (a) The system output trajectory tracks the desired trajectory exactly with the increasing iteration number (Fig. 1); (b) The convergence of the tracking error is 
achieved along the iteration direction (Fig. 2); (c) Both the system output (Fig. 1) and the control input (Fig. 3) are actively regulated to comply with the constraints.

For the purpose of comparison, the DDOILC proposed in [35] is also applied into the above trajectory tracking problem, shown as follows

$$
\begin{gathered}
\hat{\boldsymbol{\varphi}}_{k}(t)=\hat{\boldsymbol{\varphi}}_{k-1}(t)+\frac{\eta\left(\Delta y_{k-1}(t+1)-\hat{\boldsymbol{\varphi}}_{k-1}(t) \Delta \boldsymbol{u}_{k-1}(t)\right) \Delta \boldsymbol{u}_{k-1}^{T}(t)}{\mu+\left\|\Delta \boldsymbol{u}_{k-1}(t)\right\|^{2}} \\
\boldsymbol{U}_{k}=\boldsymbol{U}_{k-1}+\frac{\rho \hat{\boldsymbol{\Phi}}_{k}^{T} \boldsymbol{E}_{k-1}}{\lambda+\left\|\hat{\boldsymbol{\Phi}}_{k}\right\|^{2}}
\end{gathered}
$$

where $\lambda>0$ and $\rho>0$ are a weighting factor and a step size factor respectively.

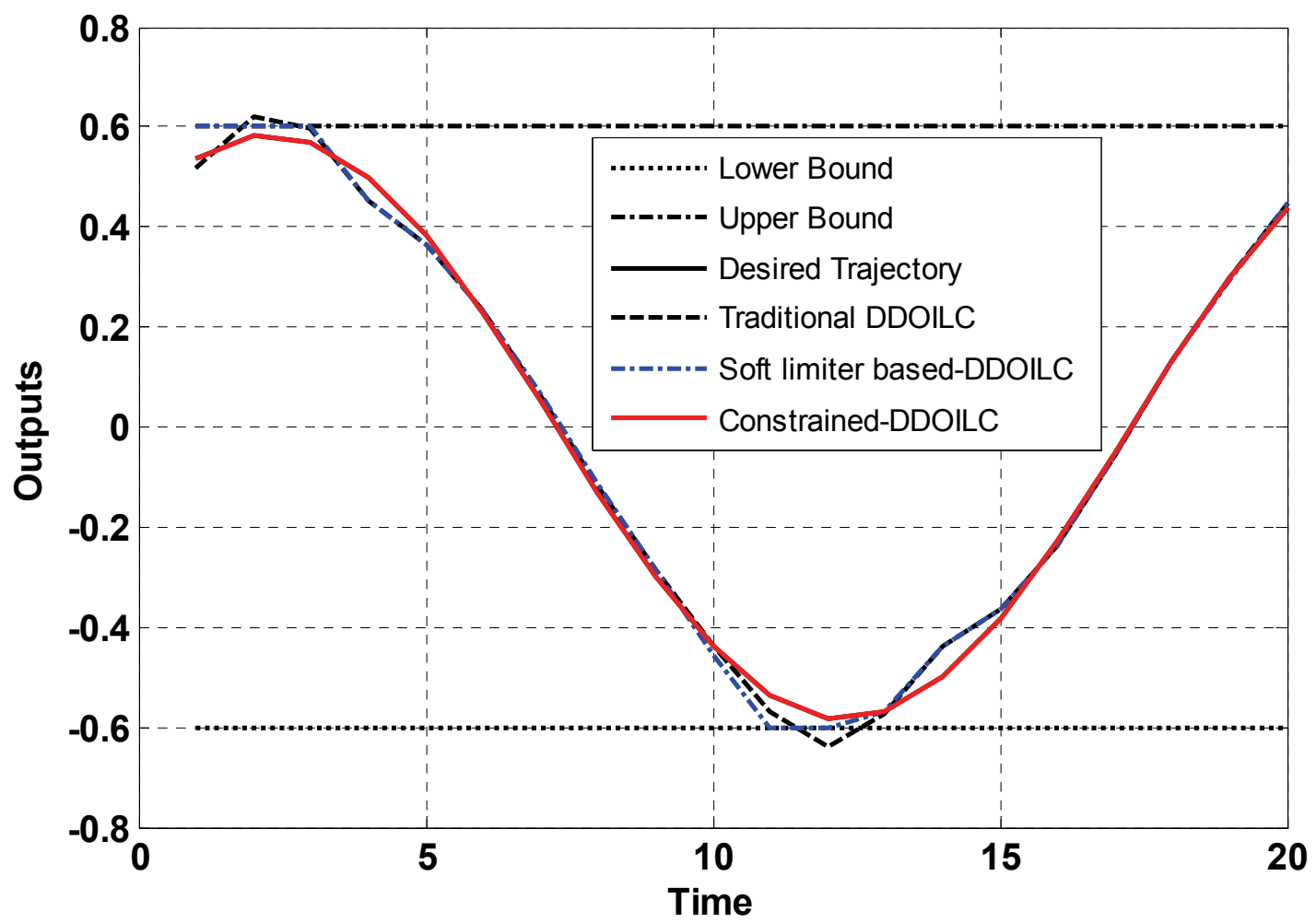

Fig. 1 The output profile at the 100-th iteration in Case 1.1 


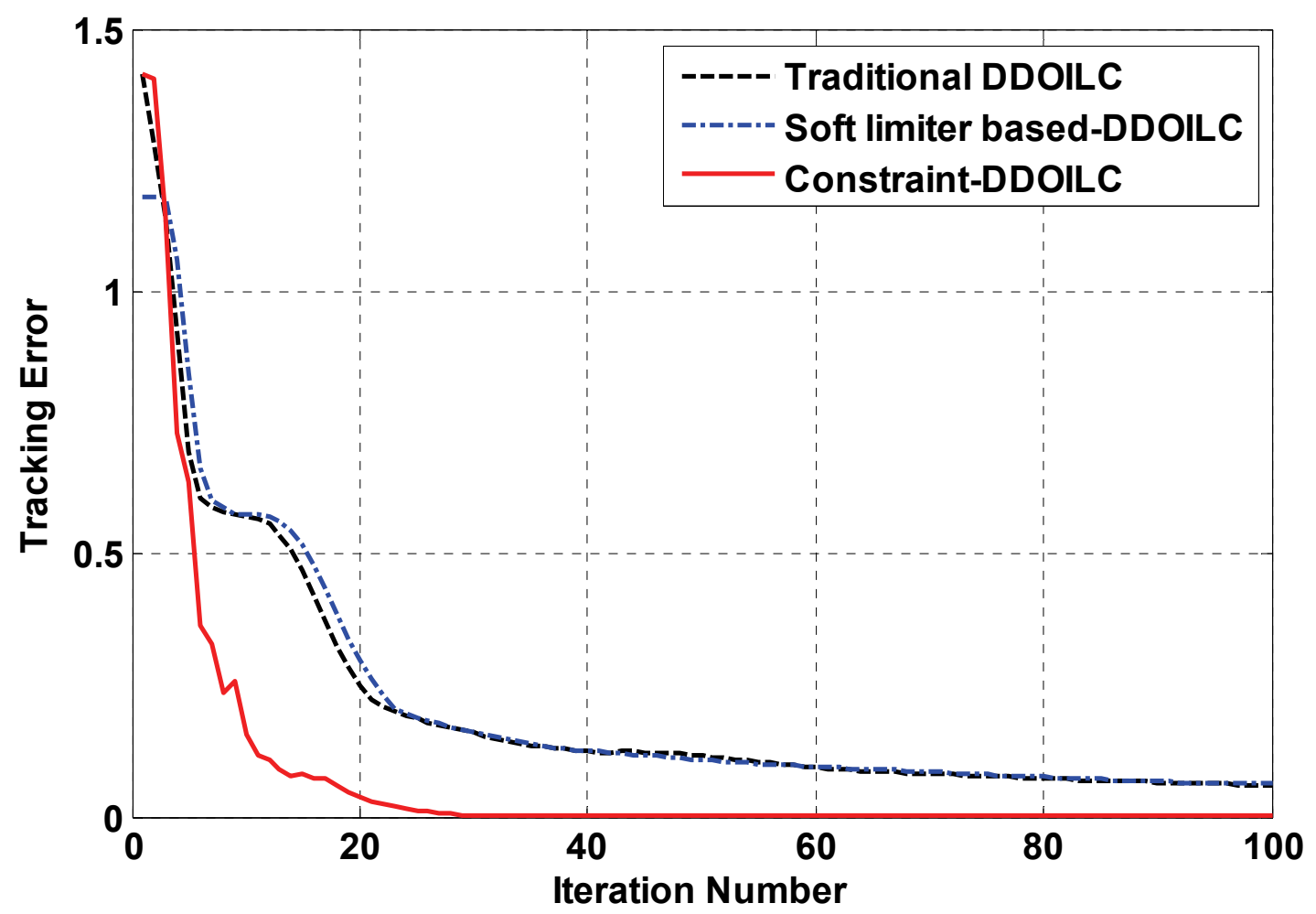

Fig. 2 The maximum absolute tracking error $e_{\max }(k)$ along with iterations in Case 1.1

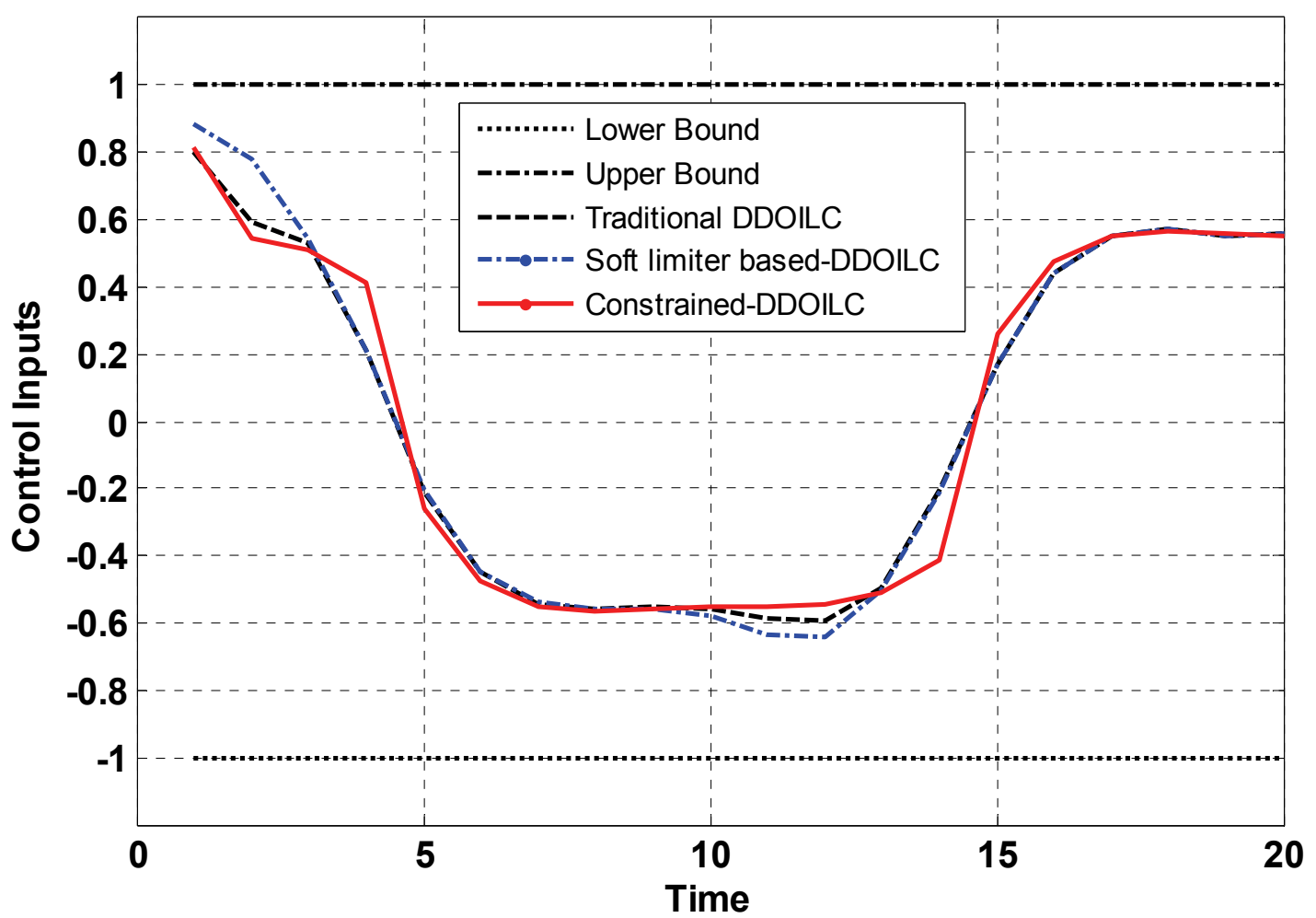

Fig. 3 The input profile at the 100-th iteration in Case 1.1 
As it is well-known, in real process industries, a soft limiter is often added directly to prevent overly large control input signals. For a further comparison, therefore, we also add a soft limiter directly to the above DDOILC with consideration of all the above system constraints. That is, the conditions of $\left|u_{k}(t)\right| \leq 1.0,\left|\Delta u_{k}(t)\right| \leq 0.25$, and $\left|y_{k}(t)\right| \leq 0.6$ are added into the calculation procedure of the DDOILC (44) - (45) as a soft limiter. We define such a control approach as soft limiter based-DDOILC in this work.

Under the same simulation background, that is, $y_{k}(0)=0, u_{0}(t)=0.5, \hat{\boldsymbol{\varphi}}_{0}(t)=0.5, \eta=1, \mu=1$ and selecting the controller parameters as $\rho=1, \lambda=0.01$, the simulation results by using the above traditional DDOILC and soft limiter based-DDOILC are shown in figures $1-3$, respectively.

The black dashed lines in figures $1-3$ denote the profiles of system output, tracking error, and the control input obtained by using the traditional DDOILC (44) - (45). Although a satisfactory convergence of tracking error can be achieved, the system output exceeds the prescribed bounds such that it cannot be realized through a practical actuator.

The blue dash-dotted lines in figures $1-3$ denote the profiles of system output, tracking error, and the control input by applying the soft limiter based-DDOILC defined above, respectively. It is seen that neither the system output nor the control input exceeds the prescribed bounds due to the soft limiter that is added in the calculation procedure directly. However, the tracking error convergence becomes worse than that of the traditional DDOILC because of the addition of the soft limiter.

Compared with both the traditional DDOILC and the soft limiter based-DDOILC methods, the proposed constrained DDOILC approach attains a better performance while neither system output nor control input violates the prescribed bounds because the I/O constraints are included into the proposed learning controller by solving a QP problem iteratively.

Case 1.2: Tracking the specified four points of the above desired trajectory. The control task is to drive the system output through the given desired points $y_{d}(4), y_{d}(9), y_{d}(14), y_{d}(20)$ of the desired trajectory (43) at time instants $\{4,9,14,20\}$ only, instead of the entire desired trajectory. The above constraints are al- 
so taken into account except that the constraints on the system outputs at the other unspecified points are not required. Applying the proposed constrained-DDOPTPILC (40) - (41) under the same simulation condition, i.e., $y_{k}(0)=0, u_{0}(t)=0.5, \hat{\boldsymbol{\varphi}}_{0}(t)=0.5, \boldsymbol{Q}=\boldsymbol{R}=I, \eta=1, \mu=1$, the simulation results are also shown in figures $4-6$.

By virtue of the simulation results, it is seen that the system output trajectory, obtained by applying the constrained-DDOPTPILC, only passes the desired points $y_{d}(4), y_{d}(9), y_{d}(14), y_{d}(20)$ precisely and the performance specification at the other points is not required (Fig. 4). And both the system output (red solid line in Fig. 4) and the control input (red solid line in Fig. 6) have been regulated to satisfy the given constraints.

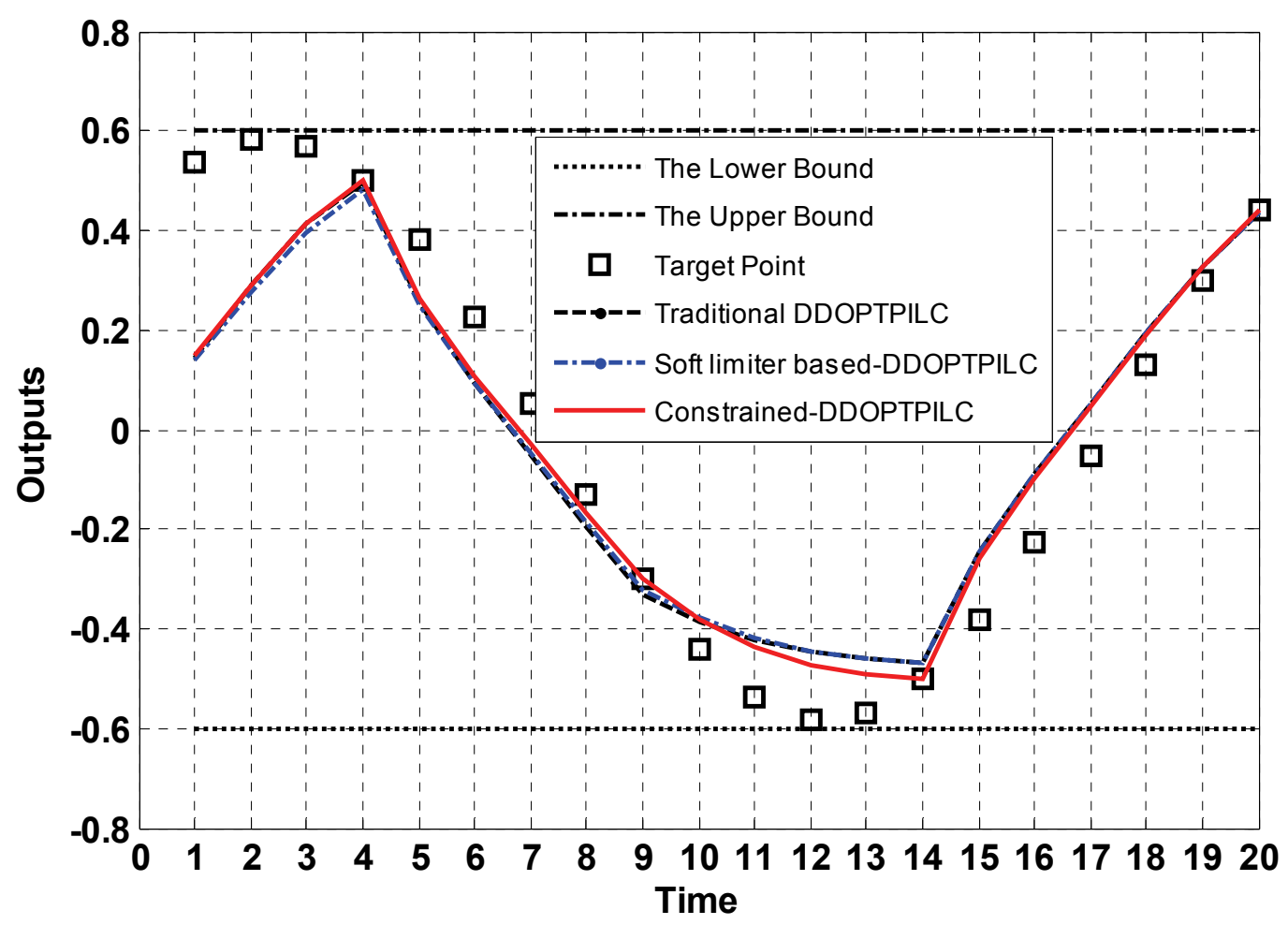

Fig. 4 The output profile at the 100-th iteration in Case 1.2 


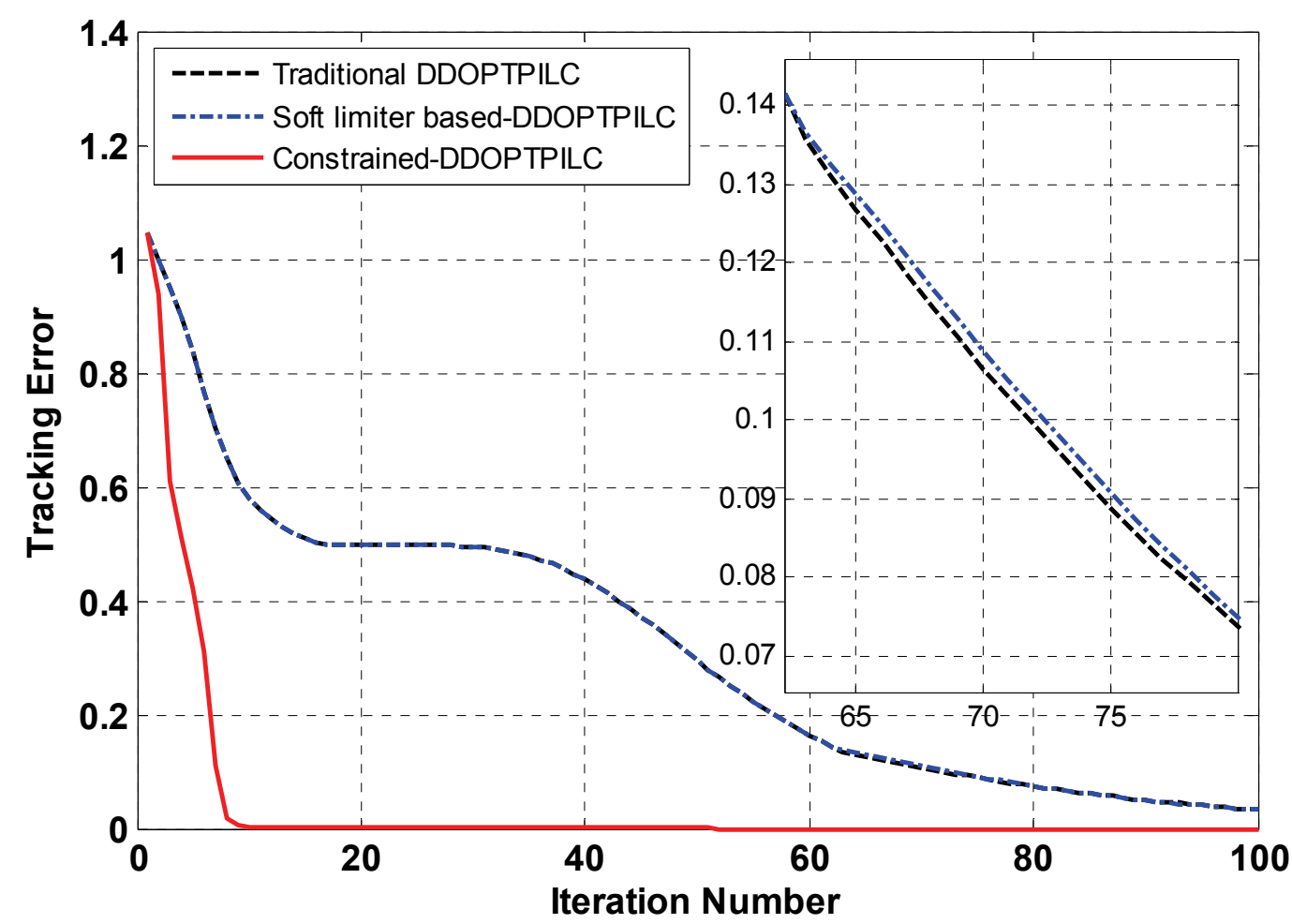

Fig. 5 The maximum absolute tracking error $e_{\max }(k)=\max _{t \in\{4,9,14,20\}}\left\{\left|e_{k}(t)\right|\right\}$ along with iterations in Case 1.2

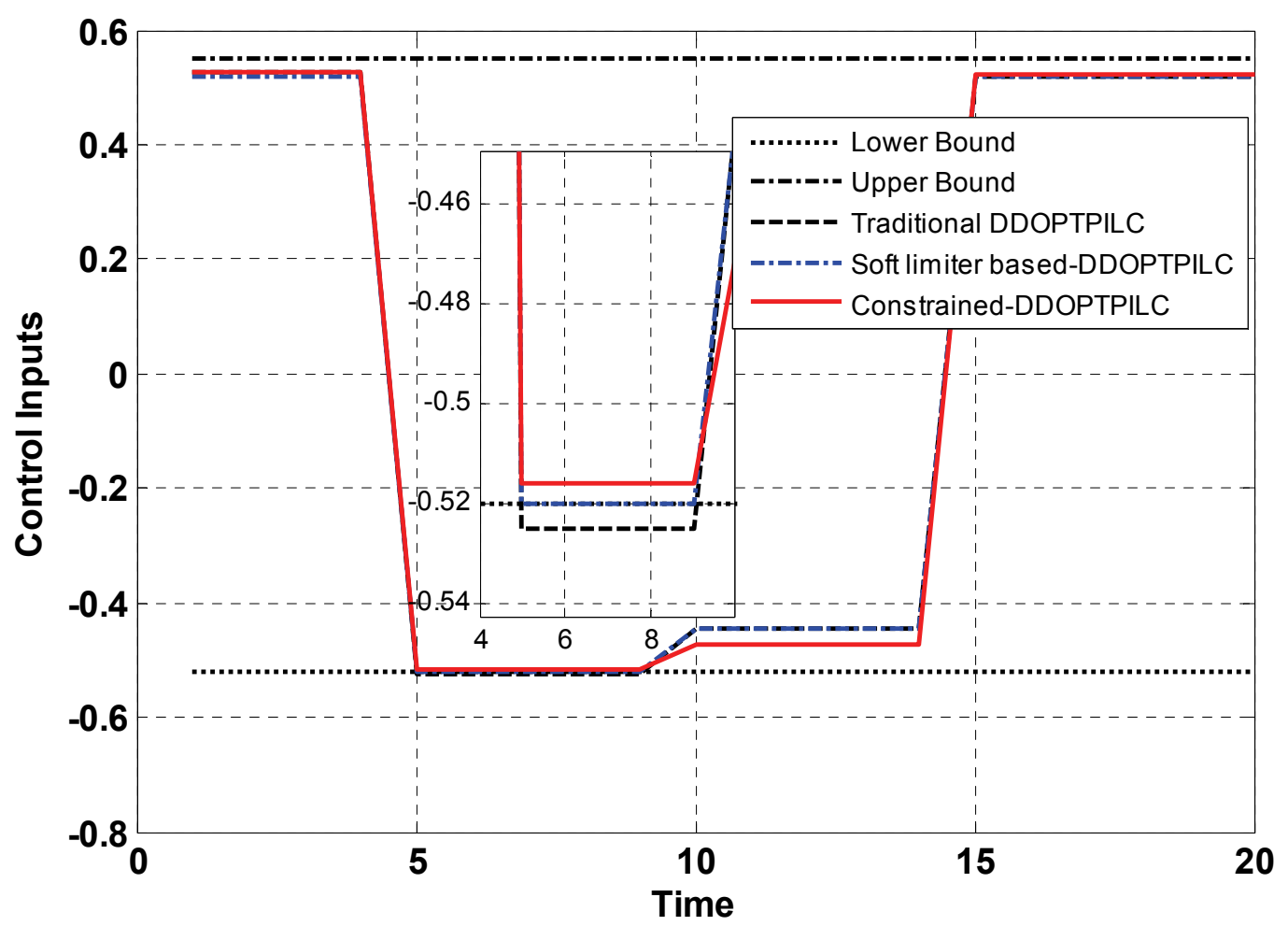

Fig. 6 The input profile at the 100-th iteration in Case 1.2 
Also, for the purpose of comparison, the traditional DDOPTPILC proposed in [35] is applied into the above trajectory tracking problem too, shown as follows

$$
\begin{gathered}
\hat{\overline{\boldsymbol{\varphi}}}_{k}\left(t_{m}-1\right)=\hat{\overline{\boldsymbol{\varphi}}}_{k-1}\left(t_{m}-1\right)+\frac{\eta\left(\Delta y_{k-1}\left(t_{m}\right)-\hat{\overline{\boldsymbol{\varphi}}}_{k-1}\left(t_{m}-1\right) \Delta \overline{\boldsymbol{u}}_{k-1}\left(t_{m}-1\right)\right) \Delta \overline{\boldsymbol{u}}_{k-1}^{T}\left(t_{m}-1\right)}{\mu+\left\|\Delta \overline{\boldsymbol{u}}_{k-1}\left(t_{m}-1\right)\right\|^{2}}, m=1, \cdots, M \\
\boldsymbol{U}_{k}=\boldsymbol{U}_{k-1}+\frac{\rho \hat{\overline{\boldsymbol{\Phi}}}_{k}^{T} \overline{\boldsymbol{E}}_{k-1}}{\lambda+\left\|\hat{\boldsymbol{\Phi}}_{k}\right\|^{2}}
\end{gathered}
$$

where $\lambda>0$ and $\rho>0$ are a weighting factor and a step size factor respectively.

A soft limiter based-DDOPTPILC is also defined by adding the constraint conditions of $\left|u_{k}(t)\right| \leq 1.0$, $\left|\Delta u_{k}(t)\right| \leq 0.25$, and $\left|y_{k}(t)\right| \leq 0.6$ into the calculation procedure of the traditional DDOPTPILC (46) - (47).

Set the same initial values and estimator parameters as $y_{k}(0)=0, u_{0}(t)=0.5, \hat{\boldsymbol{\varphi}}_{0}(t)=0.5, \eta=1, \mu=1$, and set controller parameters as $\rho=0.6, \lambda=0.1$. Figures $4-6$ show the simulation results by applying the traditional DDOPTPILC and soft limiter based-DDOPTPILC, respectively.

In terms of simulation results, the similar conclusions can be made: (1) the proposed constrained DDOPTPILC achieves the best convergence performance among the three methods, and guarantees the system output and control input being in the predesigned bounds; (2) although a satisfactory convergence of tracking error can be achieved by the traditional DDOPTPILC, its control input violates the prescribed bounds (Fig. 6); (3) soft limiter added to the calculation procedure will degrade the control performance though it can make both the control input and the system output be located within the prescribed bounds.

Case 1.3: Comparison of the proposed constrained DDOILC and constrained DDOPTPILC. As discussed in the Introduction part, the PTPILC approach may outperform ILC because many unnecessary constraints on the free points other than the specified ones are removed. To verify this standpoint, we also provide the following comparison results, shown in figures $7-9$, under the same control parameters and initial 
values for the both methods, that is, $y_{k}(0)=0, u_{0}(t)=0.5, \hat{\boldsymbol{\varphi}}_{0}(t)=0.5, \boldsymbol{Q}=\boldsymbol{R}=I, \eta=1, \mu=1$. Moreover, an index of control effort, defined as $C_{E}(k)=\left\|\boldsymbol{U}_{k}\right\|^{2}$, is introduced in this case for comparison.

Although the constrained-DDOILC can be applied to the multiple intermediate points tracking problem if all the system outputs are measurable and a desired trajectory can be designed to pass through the specified points, its control performance is inferior to that of the constrained-DDOPTPILC because more constraints on the unnecessary points should be considered to make the system output exactly track all the desired points of the reference trajectory (Fig. 7). As a result, the constrained-DDOPTPILC achieves a better control performance with faster convergence (Fig. 8) and less control effort (Fig. 9).

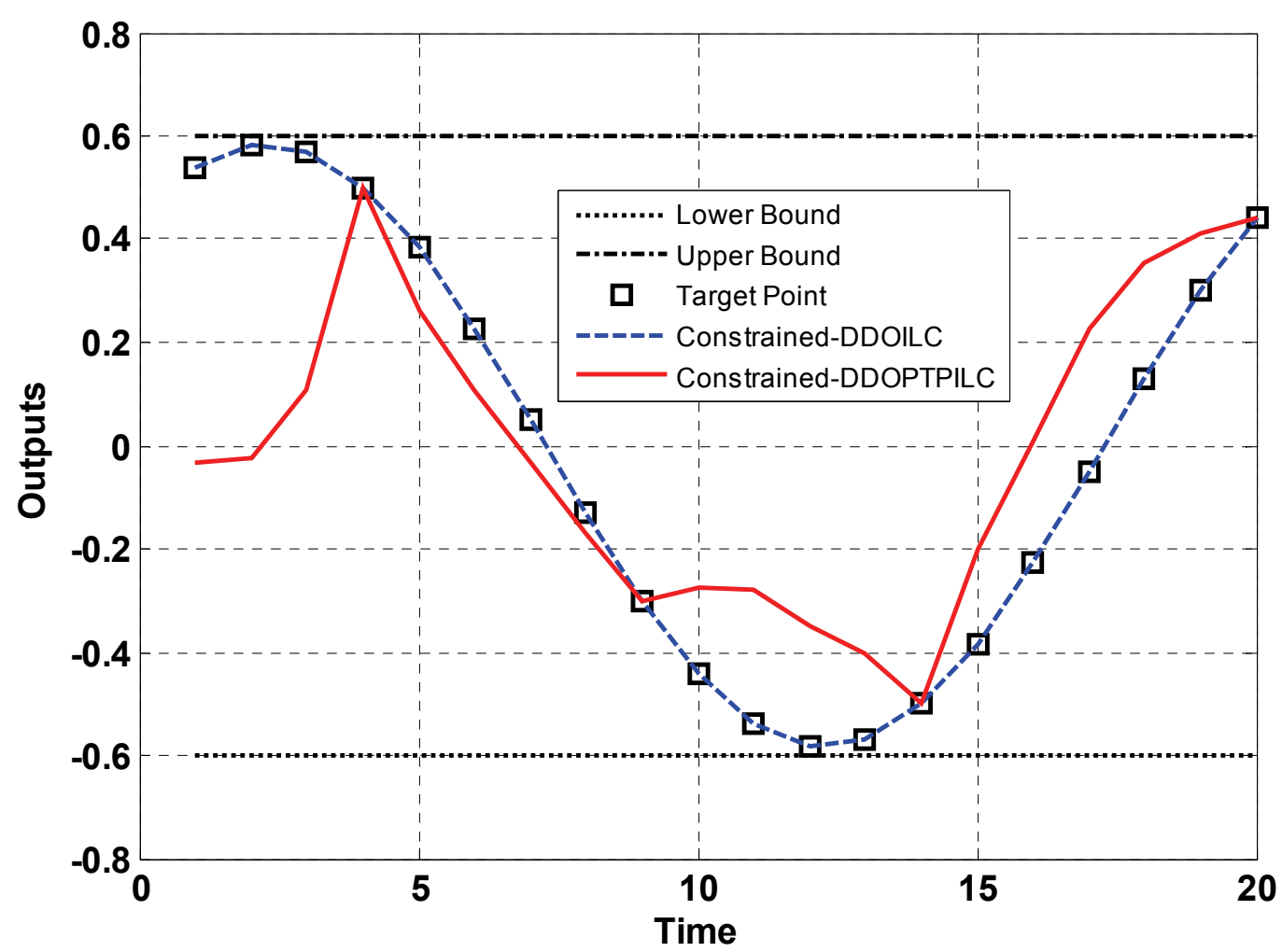

Fig. 7 The tracking performance of constrained DDOILC and constrained DDOPTPILC in example 1 


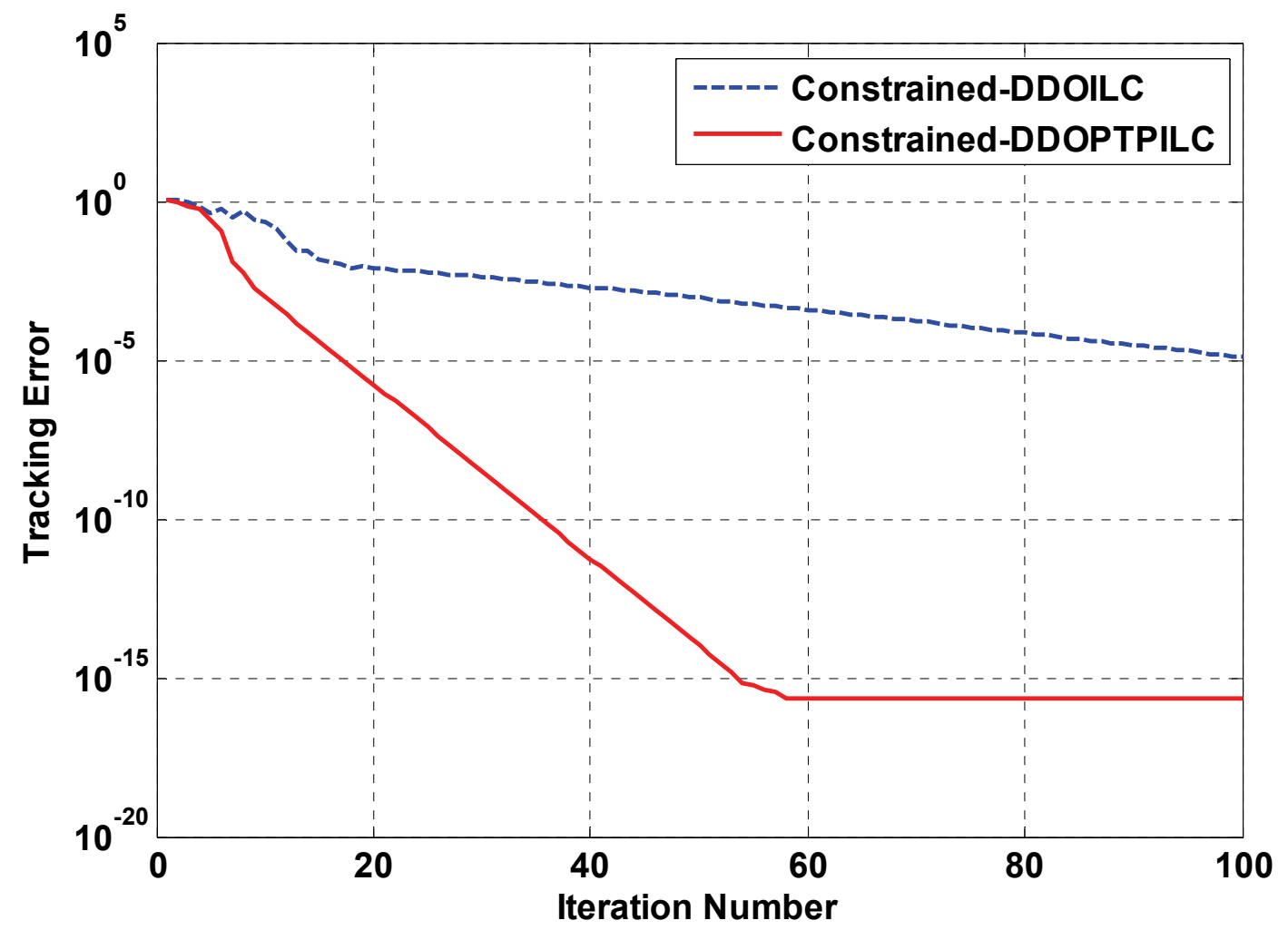

Fig. 8 The error convergence of constrained DDOILC and constrained DDOPTPILC in example 1

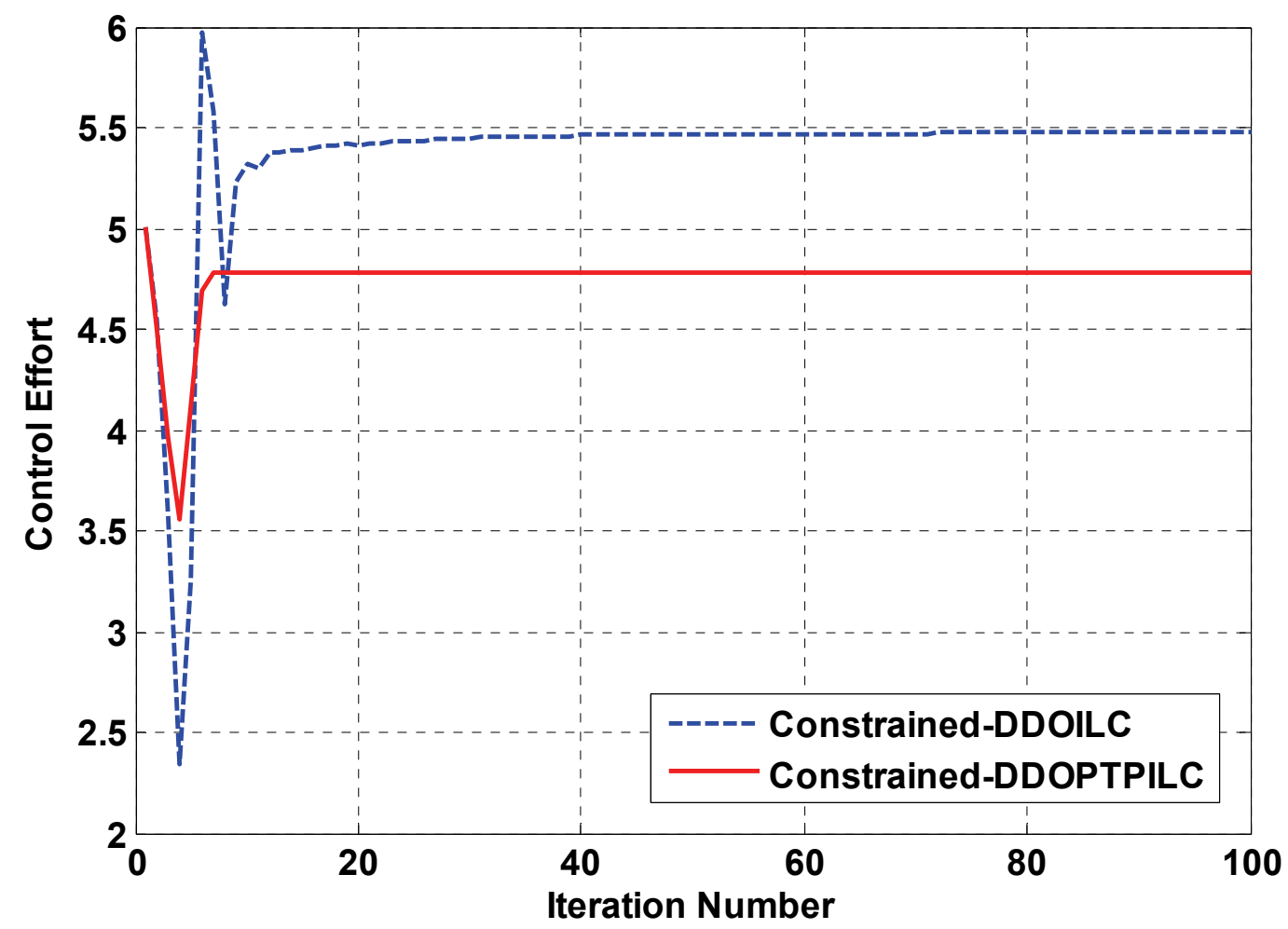

Fig. 9 The control effort of constrained DDOILC and constrained DDOPTPILC in example 1 
Case 1.4: Impact of different design parameters in the algorithms on the control performance. In general, the controller parameters play a major role in the control performance. Therefore, this case provides a detailed analysis of the parameter selection for the proposed methods, which may be useful for practical engineering applications.

First, we take the proposed constrained-DDOILC for trajectory tracking in Case 1.1 as an example to discuss the impact of the parameters in the estimator algorithm, i.e., $\eta$ and $\mu$. In terms of the estimation algorithm (21), $\mu$ exists in the denominator together with the input signals. As a result, it is difficult to clearly distinguish the effect on the control performance caused by $\mu$ or by the input signals. In general, a large $\mu$ may smooth the variation of the tracking error. In practice, we commonly fix $\mu$ with a proper value and then tune $\eta$ in its range $\{0,2\}$ to gain a satisfactory control performance. Figure 10 shows the simulation result with different values of $\eta$, where $\mu=0.1$ is fixed.

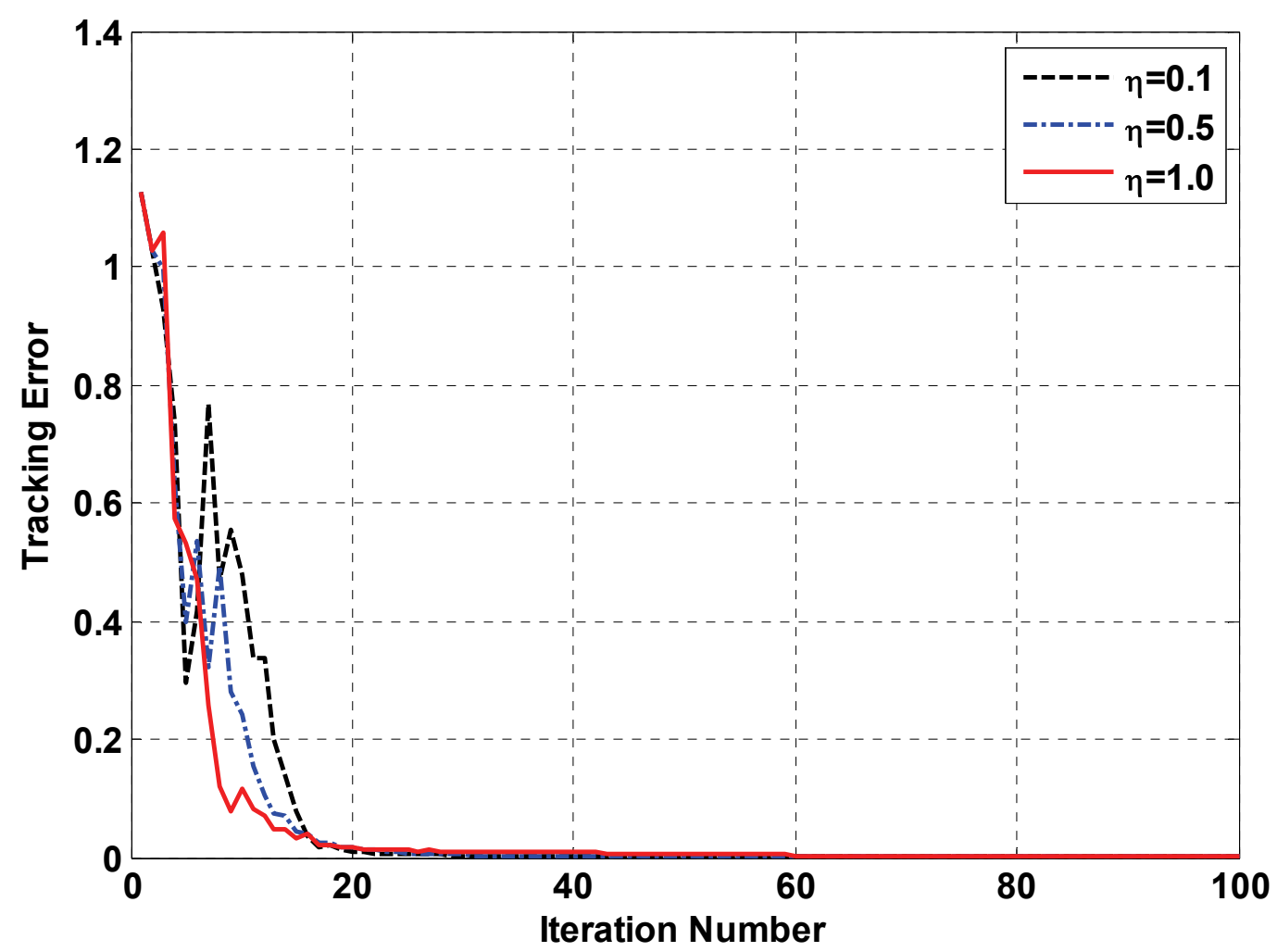

Fig. 10 The error convergence of constrained DDOILC using different values of $\eta$ 
Again from estimation algorithm (21), $\eta$ is in the numerator as a product factor. It is apparent that a larger value of $\eta$ can play a bigger role in the tracking performance. Figure 10 verifies this standpoint that a faster convergence can be achieved with a larger $\eta$. However, if the value of $\eta$ is too large, the oscillation, overshoot, and other unexpected problem may occur.

To make the comparison further, we also take the proposed constrained-DDOPTPILC as an example to show the effect of the controller parameters on the control performance. According to the index function (38), if no constraint is considered, then the control law can be solved as,

$$
\Delta \boldsymbol{U}_{k+1}=\left(\hat{\overline{\boldsymbol{\Phi}}}_{k}^{T} \boldsymbol{Q} \hat{\overline{\boldsymbol{\Phi}}}_{k}+\boldsymbol{R}\right)^{-1} \hat{\boldsymbol{\Phi}}_{k+1}^{T} \boldsymbol{Q} \overline{\boldsymbol{E}}_{k}
$$

It is apparent that $\boldsymbol{Q}$ and $\boldsymbol{R}$ are similar to the parameters of $\eta$ and $\mu$ respectively. So we commonly fix $\boldsymbol{R}$ to a proper value and then tune $\boldsymbol{Q}$ to achieve a satisfactory performance. The simulation result with different values of $\boldsymbol{Q}$, where $\boldsymbol{R}=\boldsymbol{I}$, is shown in Fig. 11, indicating that a larger $\boldsymbol{Q}$ means a faster convergence.

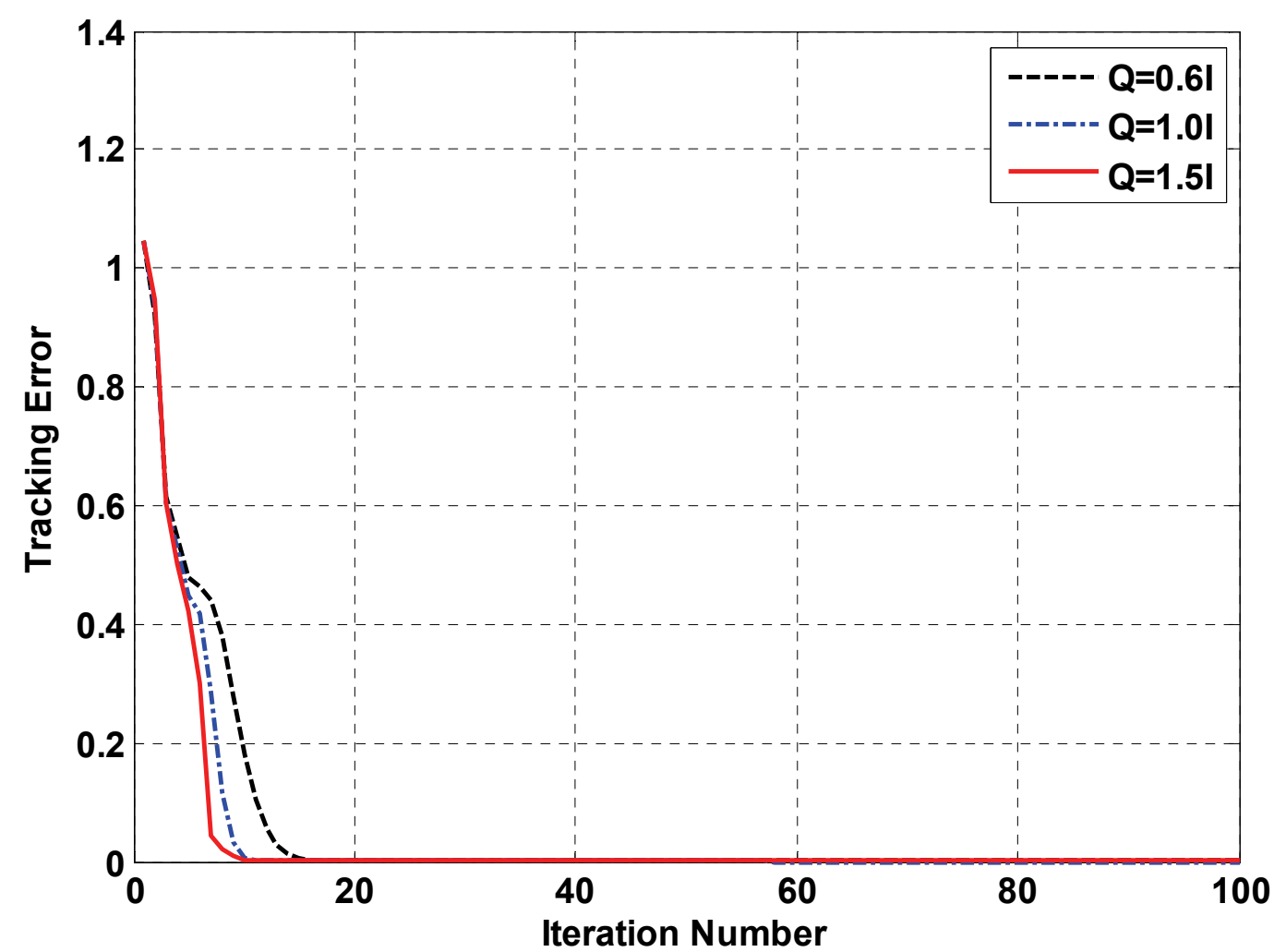

Fig. 11 The error convergence of constrained-DDOPTPILC using different values of $\boldsymbol{Q}$ 
Example 2: To show the applicability and effectiveness of the proposed approaches to realistic processes, a nonlinear batch reactor [39-41] is considered as follows,

$$
\begin{aligned}
& \frac{d x_{1}}{d t}=-k_{1} \exp \left(\frac{-E_{1}}{u T_{r e f}}\right) x_{1}^{2}+d_{k}(t) \\
& \frac{d x_{2}}{d t}=k_{1} \exp \left(\frac{-E_{1}}{u T_{r e f}}\right) x_{1}^{2}-k_{2} \exp \left(\frac{-E_{2}}{u T_{r e f}}\right) x_{2}+d_{k}(t) \\
& y=x_{2}+\delta_{k}(t)
\end{aligned}
$$

where $x_{1}$ and $x_{2}$ are two states denoting the concentrations of the raw material and the product respectively; $u=T / T_{\text {ref }}$ is the control input, where $T_{r e f}$ is the reference temperature and $T$ is the reactor temperature. The operation interval is $t \in\left[\begin{array}{ll}0 & 1.0\end{array}\right]$ hour. The sampling interval is 0.1 hour.

In the following simulation, to mimic the realistic control condition, the system disturbances $d_{k}(t)$ and output noises $\delta_{k}(t)$ are added to the batch reactor process, where both of them are randomly varying in the interval of $\left[\begin{array}{ll}-0.01 & 0.01\end{array}\right]$. The initial states are also assumed varying randomly over different iterations, that is $\boldsymbol{x}_{k}(0)=\left[x_{1, k}(0), x_{2, k}(0)\right]^{T}=\left[1+\sigma_{k}, 0\right]^{T}$, where $\sigma_{k}$ varies with iterations randomly in the interval of $\left[\begin{array}{ll}-0.1 & 0.1\end{array}\right]$

The system parameters are listed as $k_{1}=4.0 \times 10^{3}, \quad k_{2}=6.2 \times 10^{5}, \quad E_{1}=2.5 \times 10^{3} \mathrm{~K}, \quad E_{2}=5.0 \times 10^{3} \mathrm{~K}$, and $T_{r e f}=348 \mathrm{~K}$. Since the reactor temperature $T$ is limited to $298 \mathrm{~K} \leq T \leq 398 \mathrm{~K}$ and $T_{r e f}=348 \mathrm{~K}$, the control input is constrained by $298 / T_{\text {ref }} \leq u_{k}(t) \leq 336 / T_{\text {ref }}$. The other two constraints are set as $-0.01 \leq \Delta u_{k}(t) \leq 0.01$ and $-0.1 \leq y_{k}(t) \leq 0.62$.

The desired product reference trajectory is $y_{d}(t)=0.61 \sin (\pi t / 20), t \in\{0,1, \cdots, 10\}$.

It is worth pointing out that the mathematical model above has been well-tested and widely used in simulation and control as a typical industrial batch process and it can be regarded as the case of simulated practical industrial application test to show the applicability and efficiency of the proposed method [39-41]. It is also worth noting that the mathematical model of the batch reactor is nonlinear and is assumed to be una- 
vailable in the simulation, and the model simply serves as producing I/O data. Further, more random disturbances in different value-ranges are added in the following simulations to demonstrate the robustness of the proposed approach.

Case 2.1: Complete trajectory tracking. The control task is to track all points of the above desired trajectory. The initial values and controller parameters are set as $u_{0}(t)=0.88, \hat{\boldsymbol{\varphi}}_{0}(t)=0.1, \eta=1, \mu=0.2$, $\boldsymbol{Q}=\boldsymbol{I}$, and $\boldsymbol{R}=0.5 \boldsymbol{I}$. Applying the proposed constrained-DDOILC (21) - (22), the simulation results are shown as the red solid lines in figures $12-14$. Obviously, the proposed constrained-DDOILC has been verified for its applicability and effectiveness to more practical processes and achieves a satisfactory performance under random disturbances and noises (Fig. 12 and Fig. 13). In addition, both the system output and control input have been confined within the given constraints (Fig. 12 and Fig. 14).

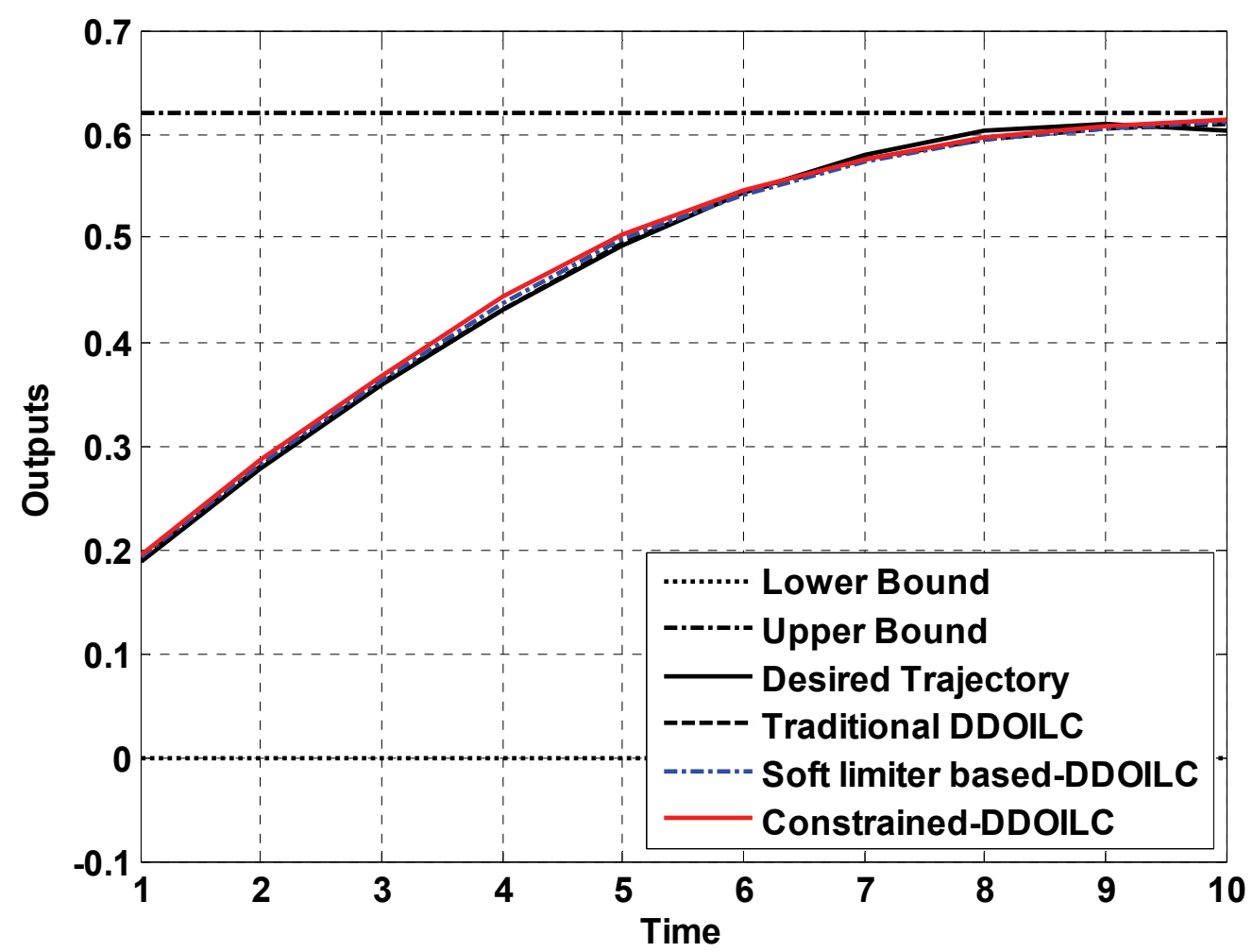

Fig. 12 The output profile at the 100-th iteration in Case 2.1 


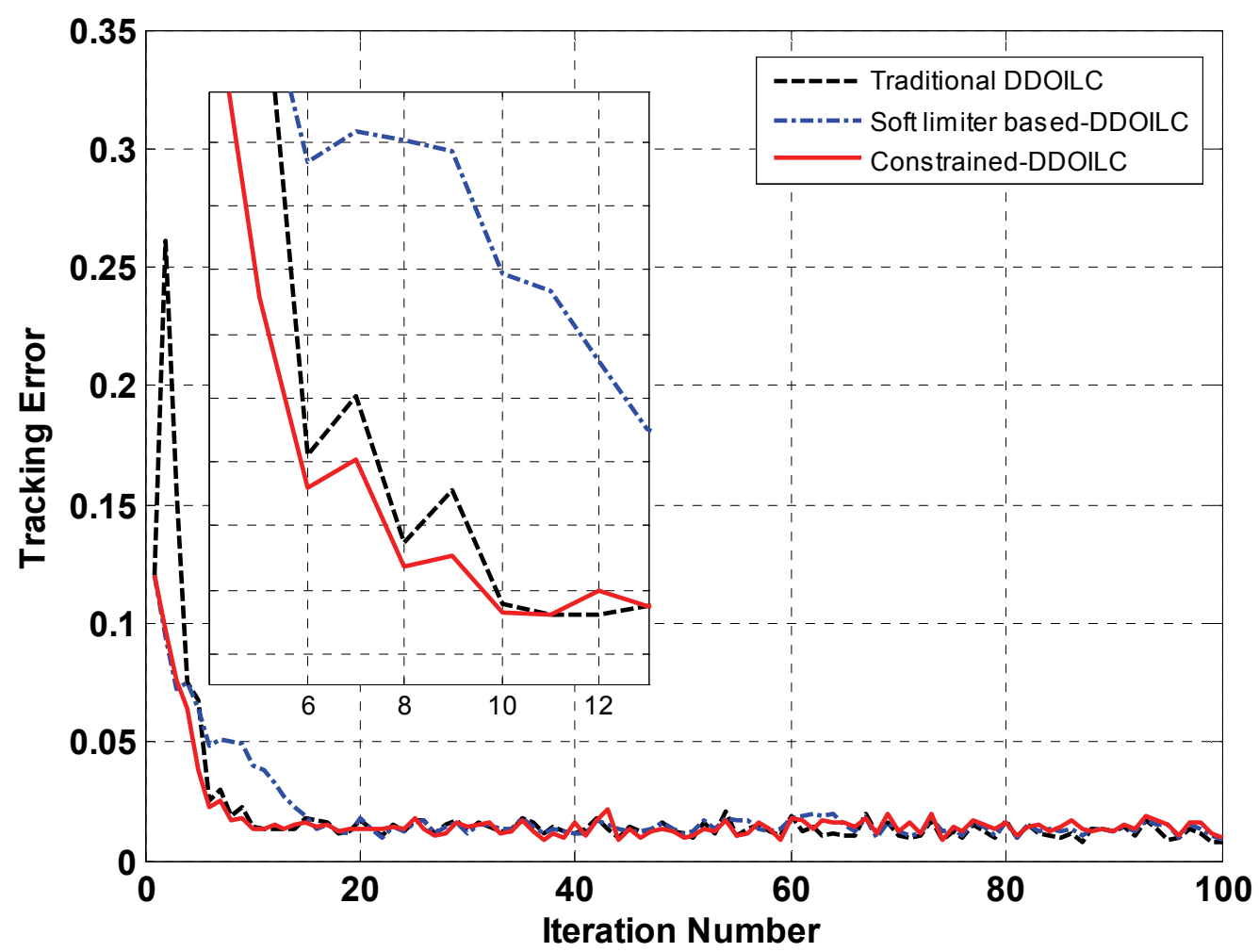

Fig. 13 The maximum absolute tracking error $e_{\max }(k)$ along with iterations in Case 2.1

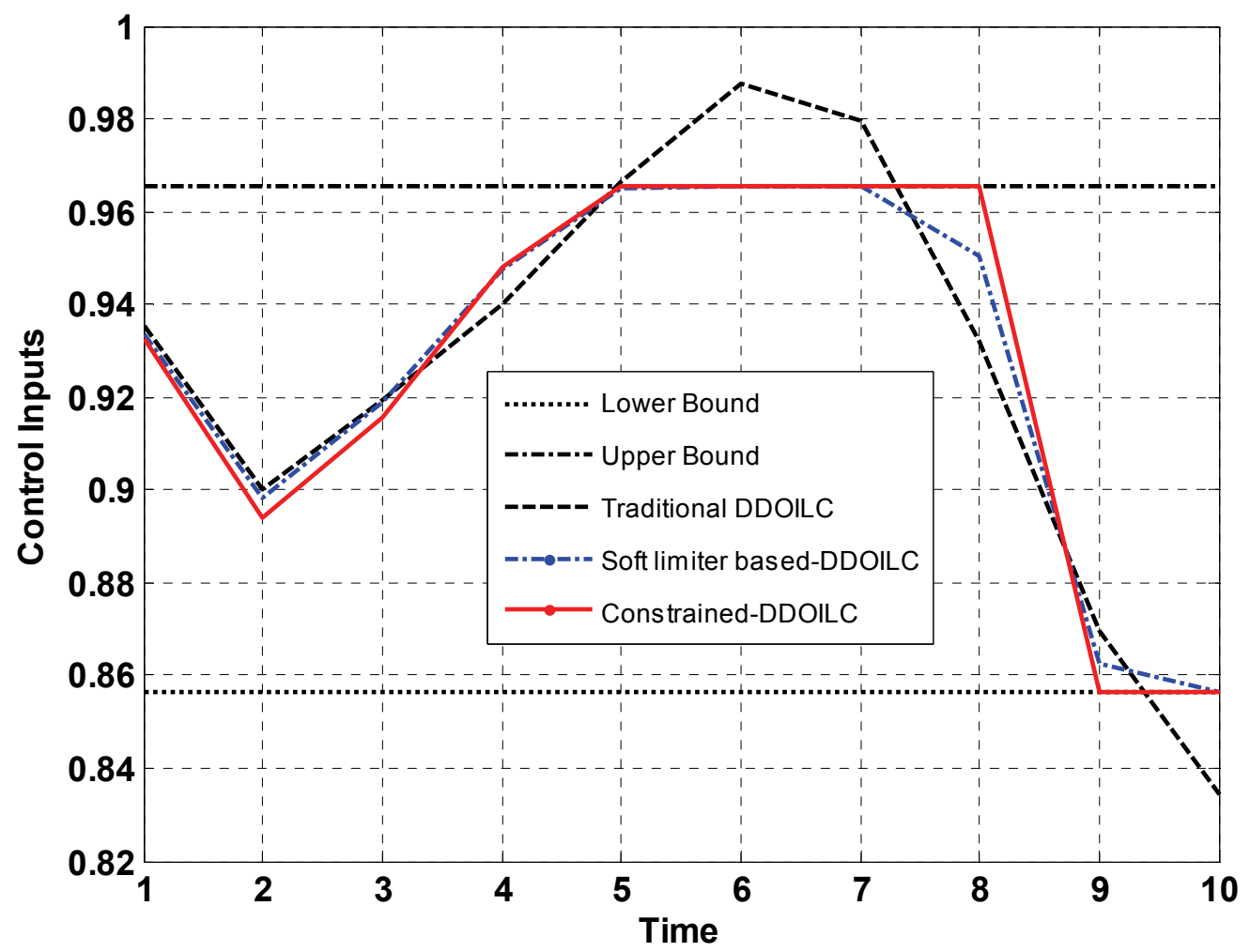

Fig. 14 The input profile at the 100-th iteration in Case 2.1 
For the comparison purpose, the traditional DDOILC (44) - (45) and the soft limiter based-DDOILC defined above are also applied to this example. Under the same simulation conditions by setting $\rho=1$, $\lambda=0.1$, the simulation results are shown as the black dashed lines and the blue dash-dotted lines in figures $12-14$, respectively.

In terms of the simulation results, it is clear that the traditional DDOILC and soft limiter based-DDOILC can also achieve a satisfactory performance. However, the control input by using traditional DDOILC goes beyond the given bounds and the soft limiter based-DDOILC reduces the convergence speed.

Case 2.2: Multiple points tracking. The control task is to track four specified points of the above trajectory at the time instants of $\{2,4,6,10\}$ only. Under the same simulation conditions as that in Case 2.1, i.e., $u_{0}(t)=0.88, \hat{\boldsymbol{\varphi}}_{0}(t)=0.1, \eta=1, \mu=0.2, \boldsymbol{Q}=\boldsymbol{I}$, and $\boldsymbol{R}=0.5 \boldsymbol{I}$, by applying the proposed constrainedDDOPTPILC (40) - (41), the simulation results are shown as the red solid lines in figures $15-17$.

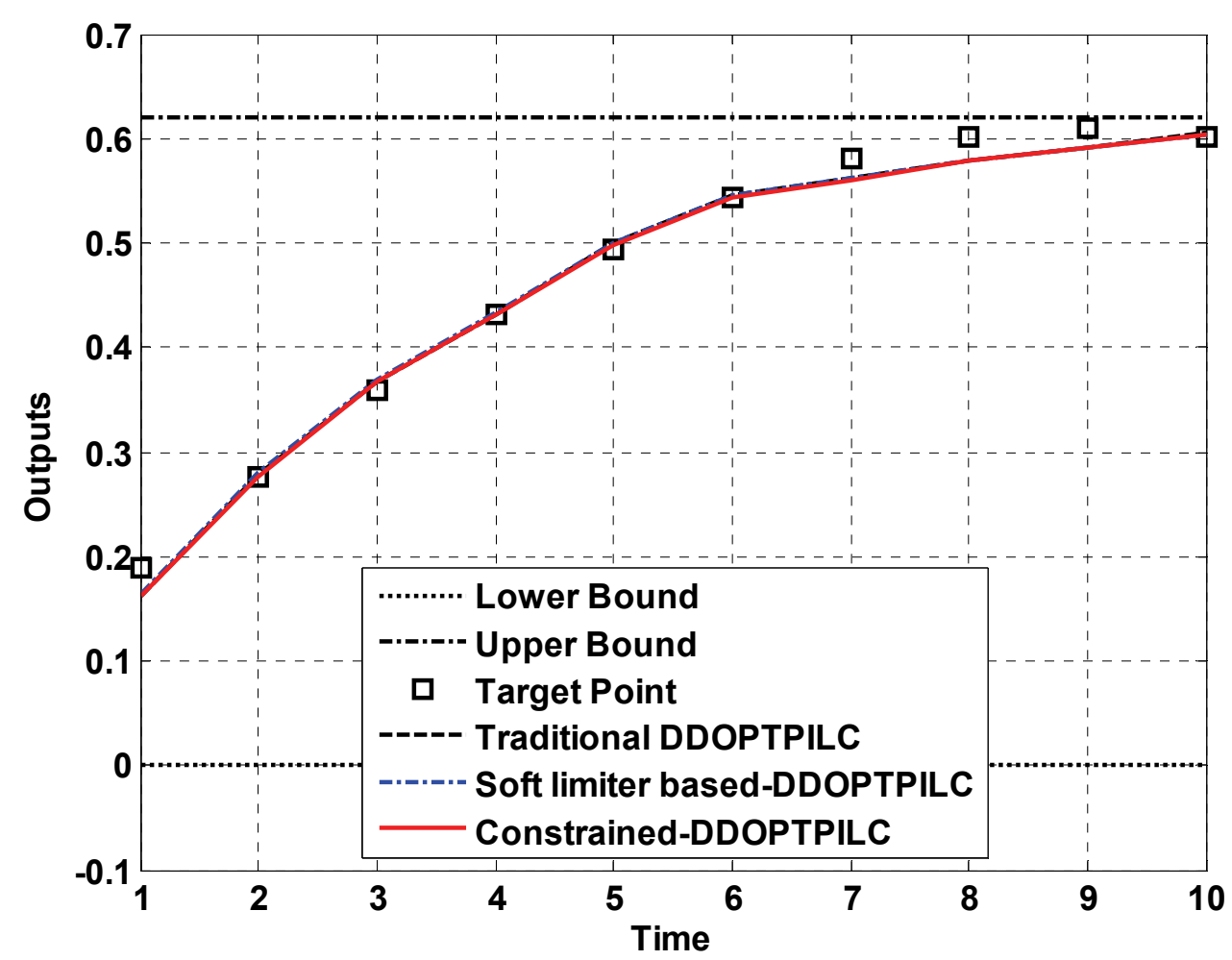

Fig. 15 The output profile at the 100-th iteration in Case 2.2 


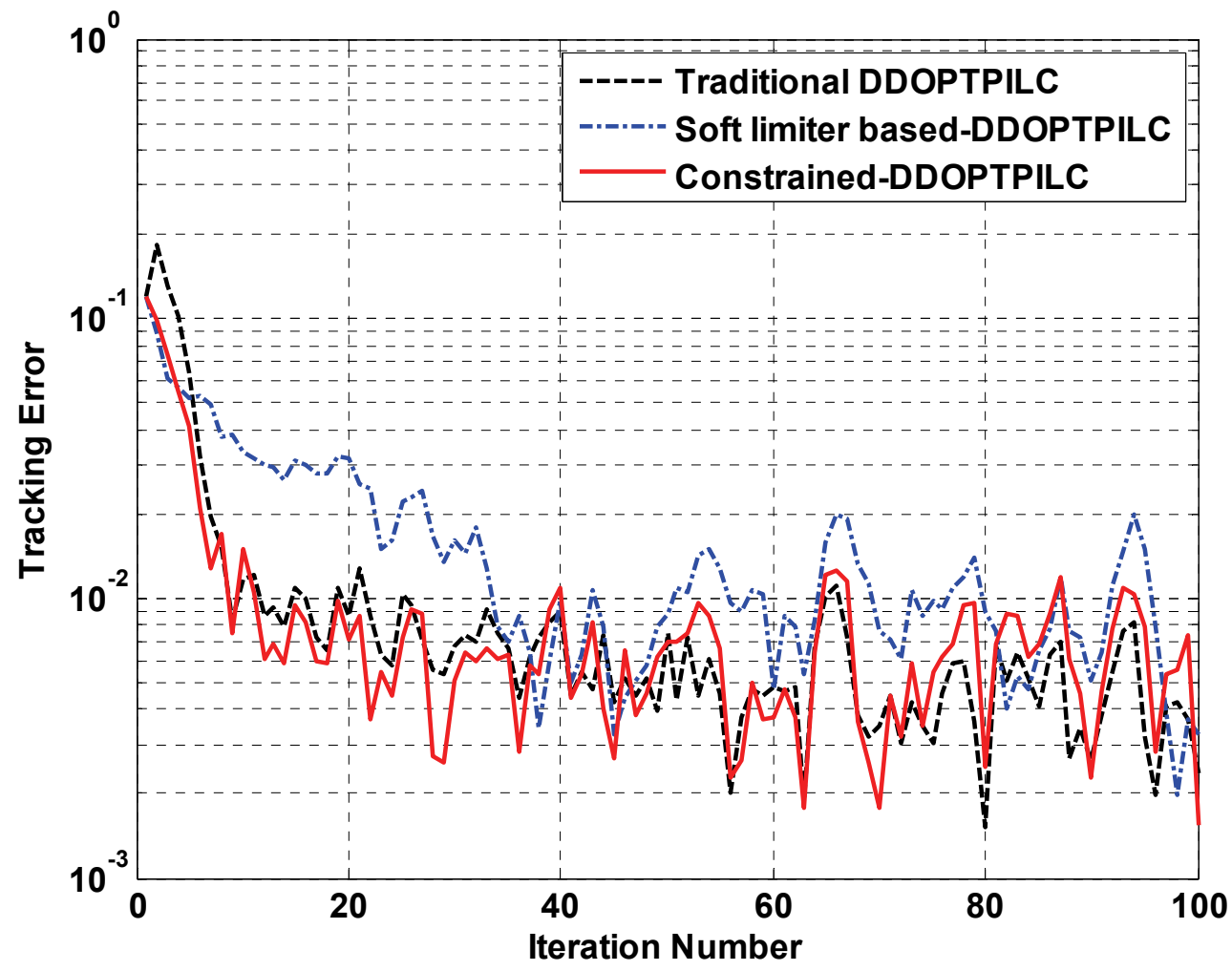

Fig. 16 The maximum absolute tracking error $e_{\max }(k)=\max _{t \in\{4,9,14,20\}}\left\{\left|e_{k}(t)\right|\right\}$ along with iterations in Case 2.2

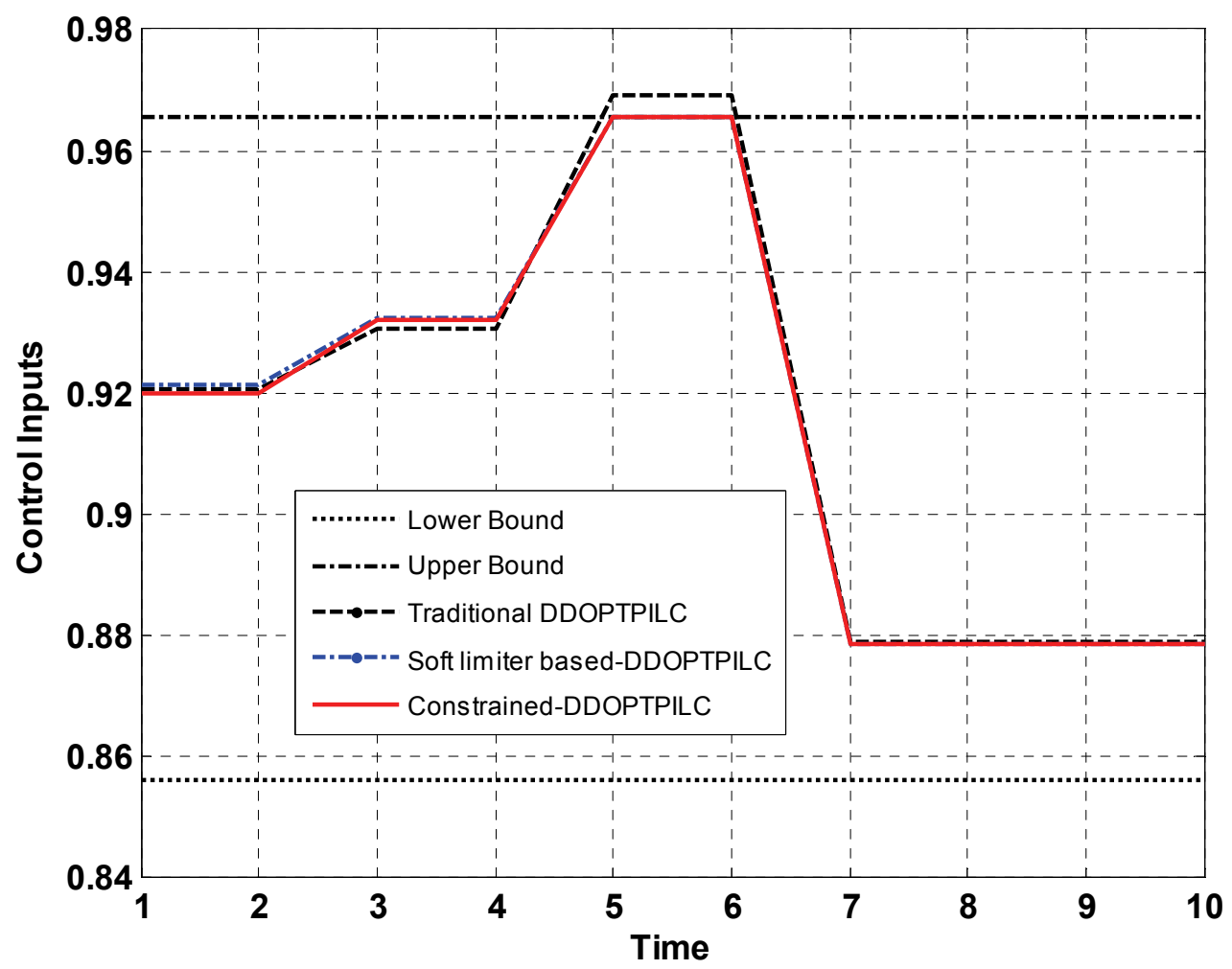

Fig. 17 The input profile at the 100-th iteration in Case 2.2 
It is shown that the proposed constrained-DDOPTPILC is effective in dealing with constrained processes with a good performance under random disturbances and noises. Both the system output and control input are constrained within the given bounds.

Applying the traditional DDOPTPILC and the soft limiter based-DDOPTPILC under the same simulation condition for the comparison, the simulation results are also shown in figures $15-17$. Fig. 17 illustrates that the control input violates the prescribed bounds by using traditional DDOPTPILC. Fig. 16 together with Fig. 17 indicates that the soft limiter based-DDOPTPILC can deal with the constrained processes, but the control performance is degraded as a trade-off.

Case 2.3: Performance comparison of the constrained-DDOILC with constrained-DDOPTPILC. In this case, a performance comparison is also provided between the constrained DDOILC and constrained DDOPTPILC when both of them are applied to the realistic nonlinear batch reactor for the multiple points tracking, where the constrained-DDOILC aims to track all the points of the given desired discrete-time trajectory $y_{d}(t)=0.61 \sin (\pi t / 20)$ which exactly passes through the four points at the time instants of $\{2,4,6,10\}$. The simulations results are shown in figures $18-20$ under the same simulation condition, i.e., $u_{0}(t)=0.88, \hat{\boldsymbol{\varphi}}_{0}(t)=0.1, \eta=1, \mu=0.2, \boldsymbol{Q}=\boldsymbol{I}$, and $\boldsymbol{R}=0.5 \boldsymbol{I}$. From Fig. 18, the system output can exactly track all the desired points by using the constrained-DDOILC, while the constrained-DDOPTPILC only makes the system output track the prescribed points at $\{2,4,6,10\}$ precisely. Figures 19 and 20 also confirm that the constrained-DDOPTPILC outperforms the constrained-DDOILC for multiple points tracking tasks with a faster convergence and reduced control effort.

Case 2.4: The robustness of the proposed constrained methods. To show the robustness of the proposed methods more extensively in dealing with exogenous uncertainties, the simulation results are further provided under different levels of stochastic disturbances. Fig. 21 shows the error convergence of the constrained-DDOILC for the complete trajectory tracking in Case 2.1. Fig. 22 shows the error convergence of the constrained-DDOPTPILC for the multiple points tracking in Case 2.2. 


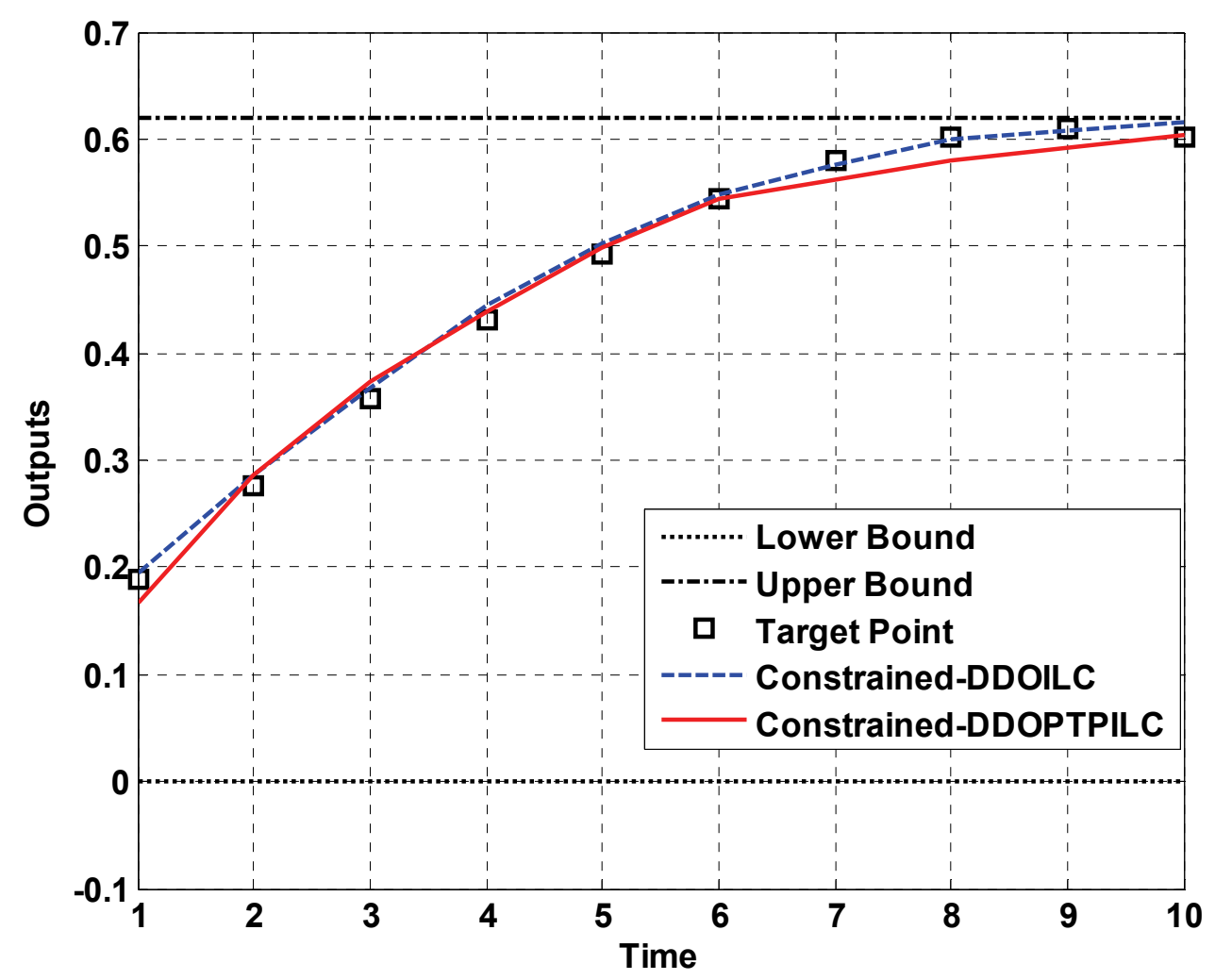

Fig. 18 The tracking performance of constrained-DDOILC and constrained-DDOPTPILC in example 2

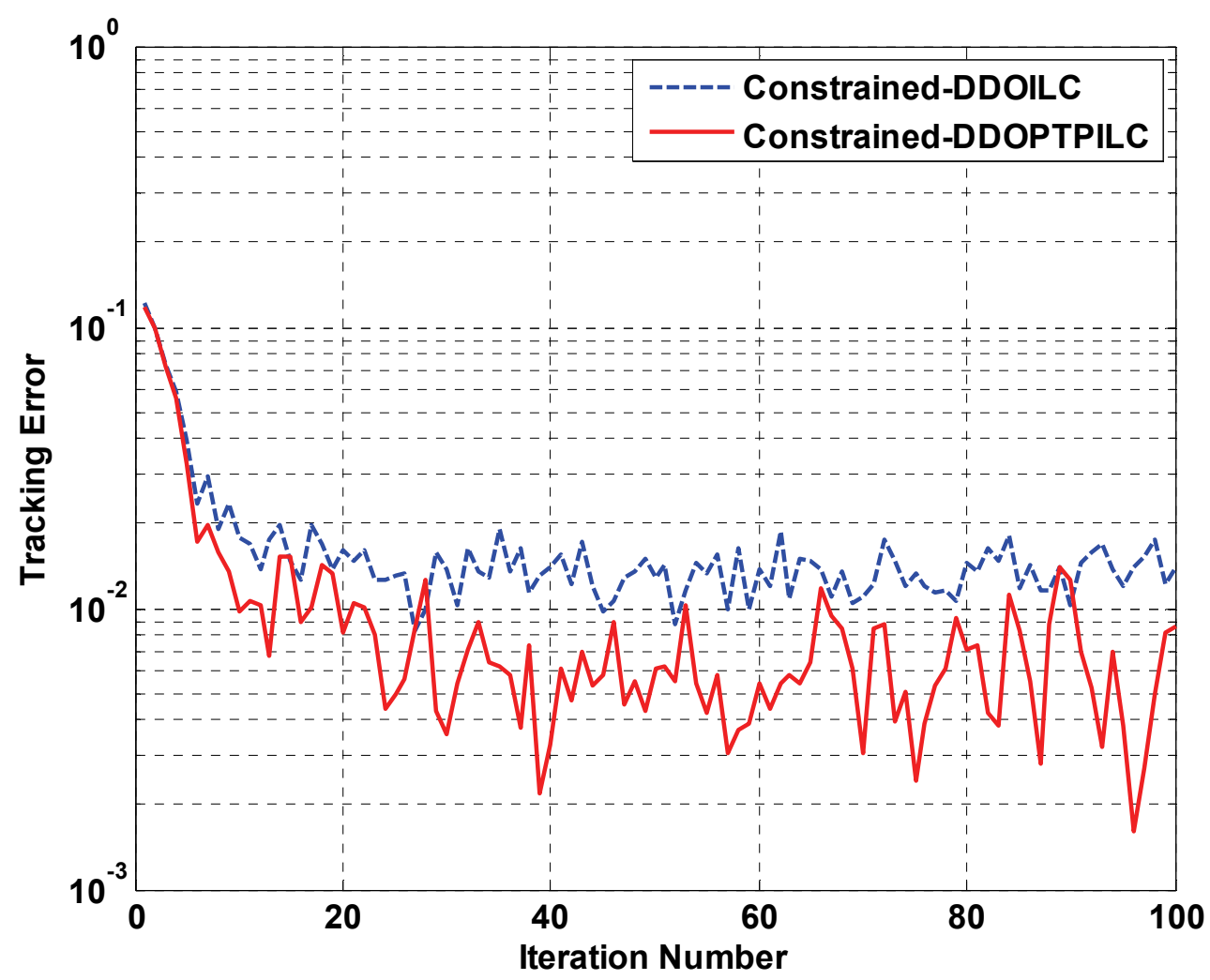

Fig. 19 The error convergence of constrained DDOILC and constrained DDOPTPILC in example 2 


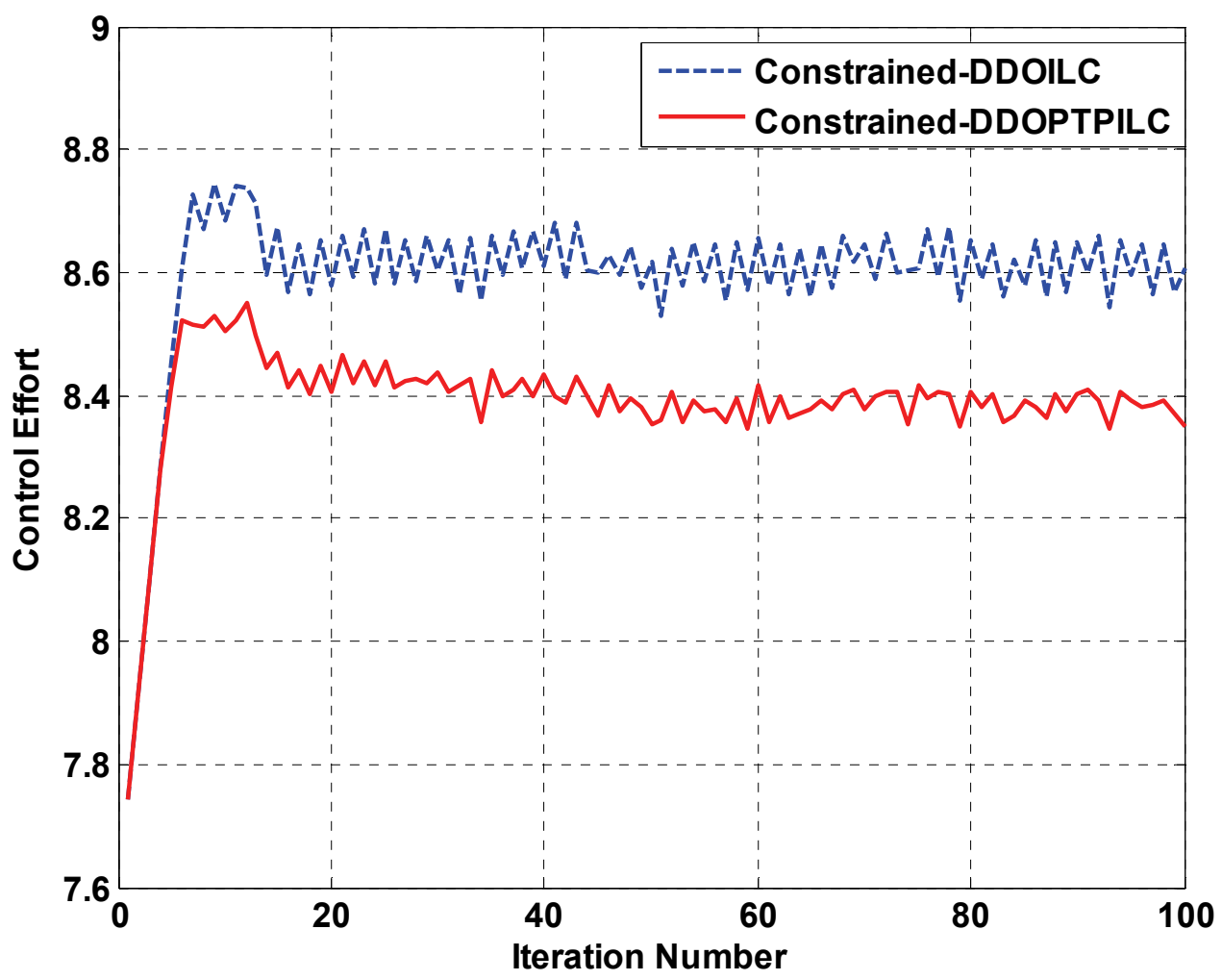

Fig. 20 The control effort of constrained DDOILC and constrained DDOPTPILC in example 2

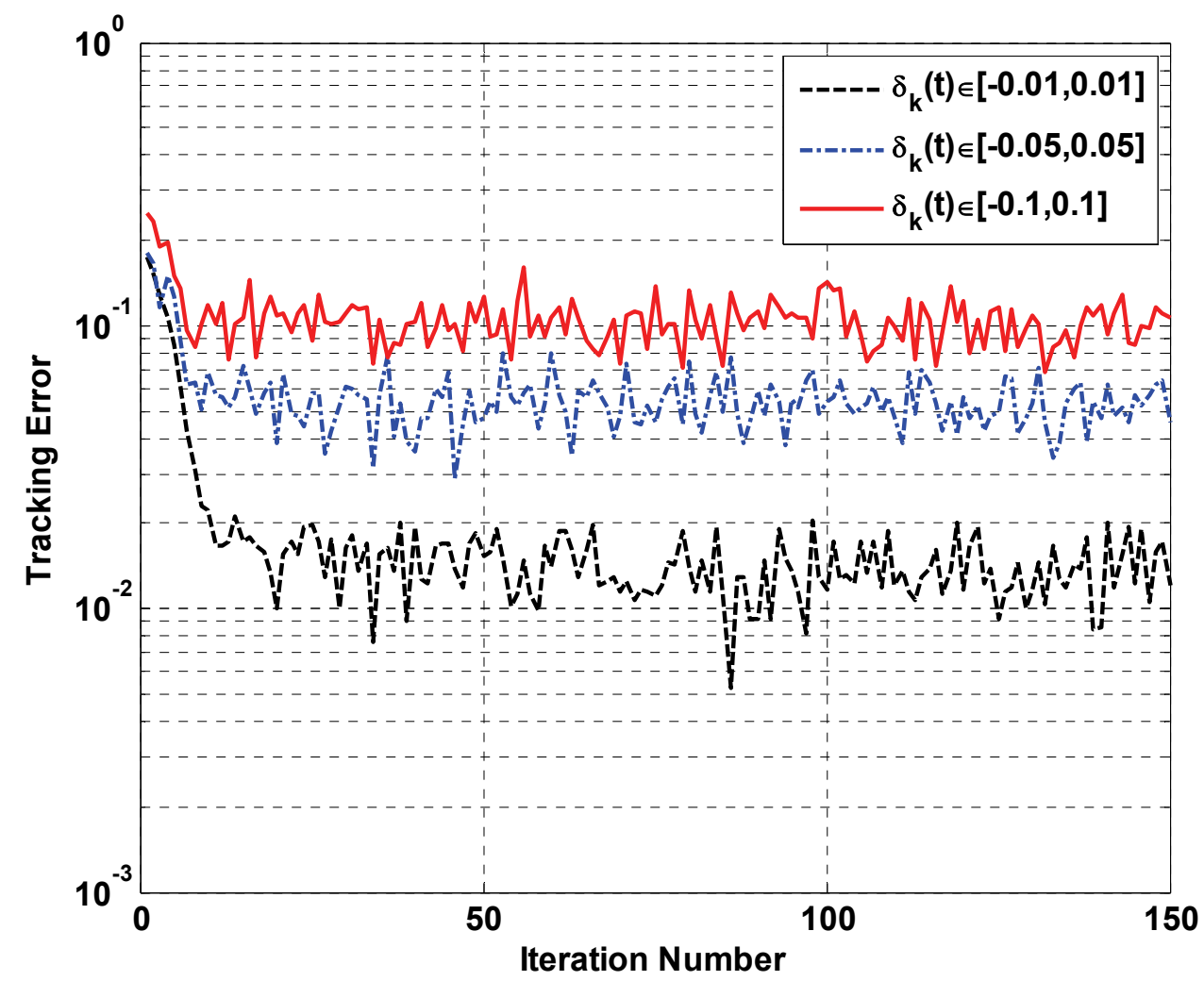

Fig. 21 The robustness of the constrained-DDOILC under different disturbances 


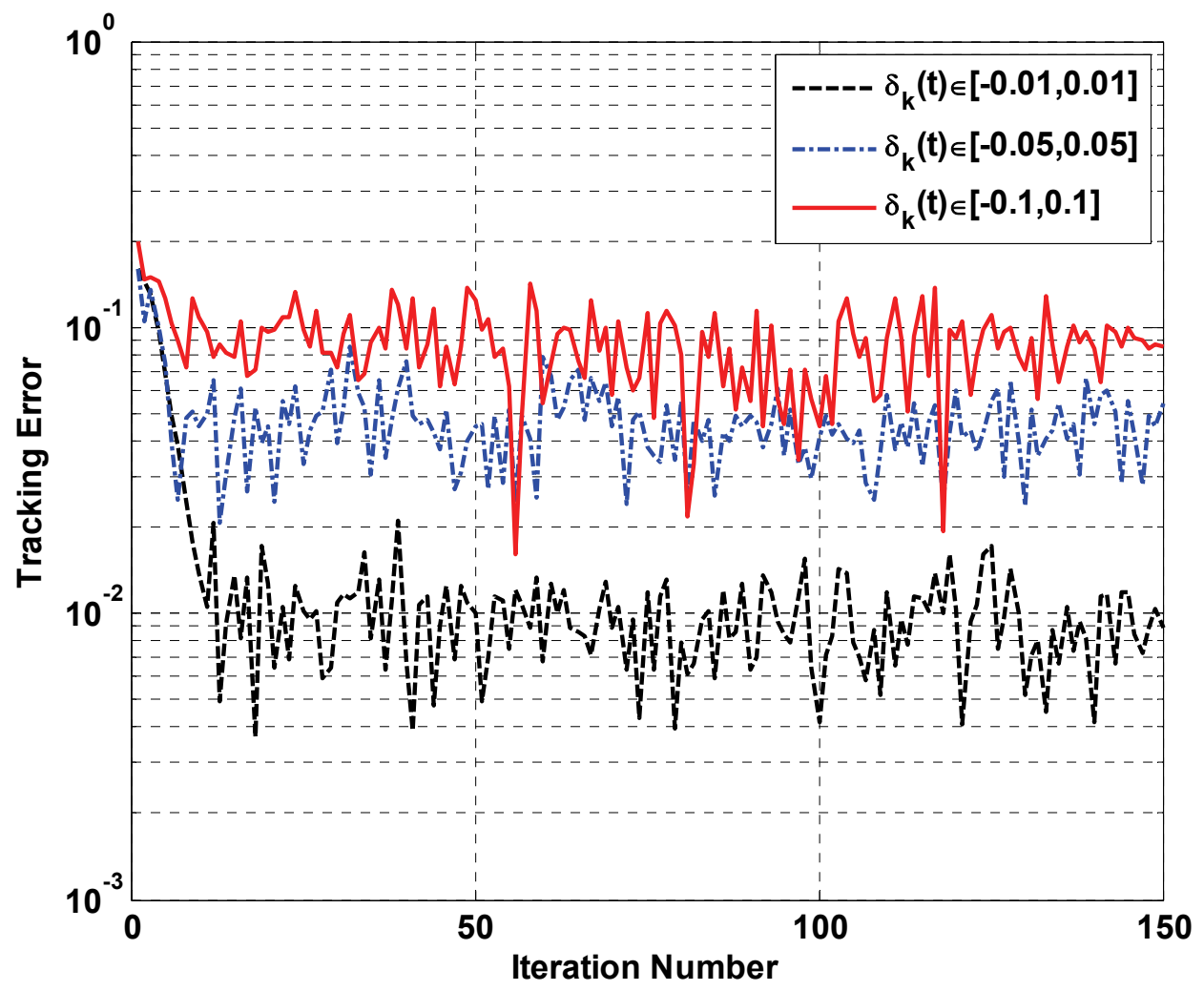

Fig. 22 The robustness of the constrained-DDOPTPILC under different disturbances

In view of figures 21 and 22, one can conclude that the proposed constrained methods in this work are capable of dealing with the exogenous bounded uncertainties.

Example 3. It is worth pointing out that data-driven control approach does not mean that the modelbased control approaches should be excluded; instead, both have their special features and complement each other. If the accurate linear model is available, the model-based control approach may achieve a better performance by using the known model information fully than the data-driven approach where no model information is used even though such information may be known as a priori. Therefore, for the purpose of comparison with the model-based methods, a slightly modified linear discrete-time system is selected from [15]

$$
\left\{\begin{array}{l}
\boldsymbol{x}(t+1)=\boldsymbol{A x}(t)+\boldsymbol{B u}(t) \\
\boldsymbol{y}(t)=\boldsymbol{C x}(t)
\end{array}\right.
$$

where $\boldsymbol{A}=\left[\begin{array}{cc}0 & 1 \\ -0.5 & -0.5\end{array}\right], \boldsymbol{B}=\left[\begin{array}{l}0 \\ 1\end{array}\right]$, and $\boldsymbol{C}=\left[\begin{array}{cc}1 & 0\end{array}\right] ; t \in\{0, \cdots, 20\}$. The desired trajectory is 


$$
y_{d}(t)=10^{-6}(t-1)^{3}(4-0.03(t-1)), t \in\{0, \cdots, 20\}
$$

And a traditional constrained norm optimal ILC law, shown as follows, is applied

$$
\left\{\begin{array}{l}
\min _{\boldsymbol{U}_{k+1}} J\left(\boldsymbol{E}_{k+1}, \boldsymbol{U}_{k+1}\right)=\min _{\boldsymbol{U}_{k+1}} \frac{1}{2}\left\{\Delta \boldsymbol{U}_{k+1}^{T}\left(\boldsymbol{G}^{T} \boldsymbol{Q} \boldsymbol{G}+\boldsymbol{R}\right) \Delta \boldsymbol{U}_{k+1}-2 \boldsymbol{E}_{k}^{T} \boldsymbol{Q} \boldsymbol{G} \Delta \boldsymbol{U}_{k+1}+\boldsymbol{E}_{k}^{T} \boldsymbol{Q} \boldsymbol{E}_{k}\right\} \\
\text { s.t. } \quad \boldsymbol{A}_{k} \Delta \boldsymbol{U}_{k+1} \leq \boldsymbol{b}_{k}
\end{array}\right.
$$

where $\boldsymbol{A}_{k}=\left[\begin{array}{c}\boldsymbol{I} \\ -\boldsymbol{I} \\ \boldsymbol{G} \\ -\boldsymbol{G}\end{array}\right], \quad \boldsymbol{b}_{k}=\left[\begin{array}{c}\Delta \boldsymbol{U}_{k}^{h i^{*}} \\ -\Delta \boldsymbol{U}_{k}^{l o w^{*}} \\ \boldsymbol{Y}^{h i}-\boldsymbol{Y}_{k} \\ -\boldsymbol{Y}^{l o w}+\boldsymbol{Y}_{k}\end{array}\right], \boldsymbol{G}=\left[\begin{array}{cccc}\boldsymbol{C B} & 0 & \cdots & 0 \\ \boldsymbol{C} \boldsymbol{B} \boldsymbol{B} & \boldsymbol{C B} & \cdots & 0 \\ \vdots & \vdots & \ddots & \vdots \\ \boldsymbol{C} \boldsymbol{A}^{N-1} \boldsymbol{B} & \boldsymbol{C} \boldsymbol{A}^{N-2} \boldsymbol{B} & \cdots & \boldsymbol{C B}\end{array}\right] . \boldsymbol{U}_{k}$ and $\boldsymbol{E}_{k}$ are two supervectors of the control input and tracking error respectively; $\boldsymbol{Q}$ and $\boldsymbol{R}$ are real symmetric positive definite matrices with appropriate dimensions; $N=20$ is the terminal point of the finite interval.

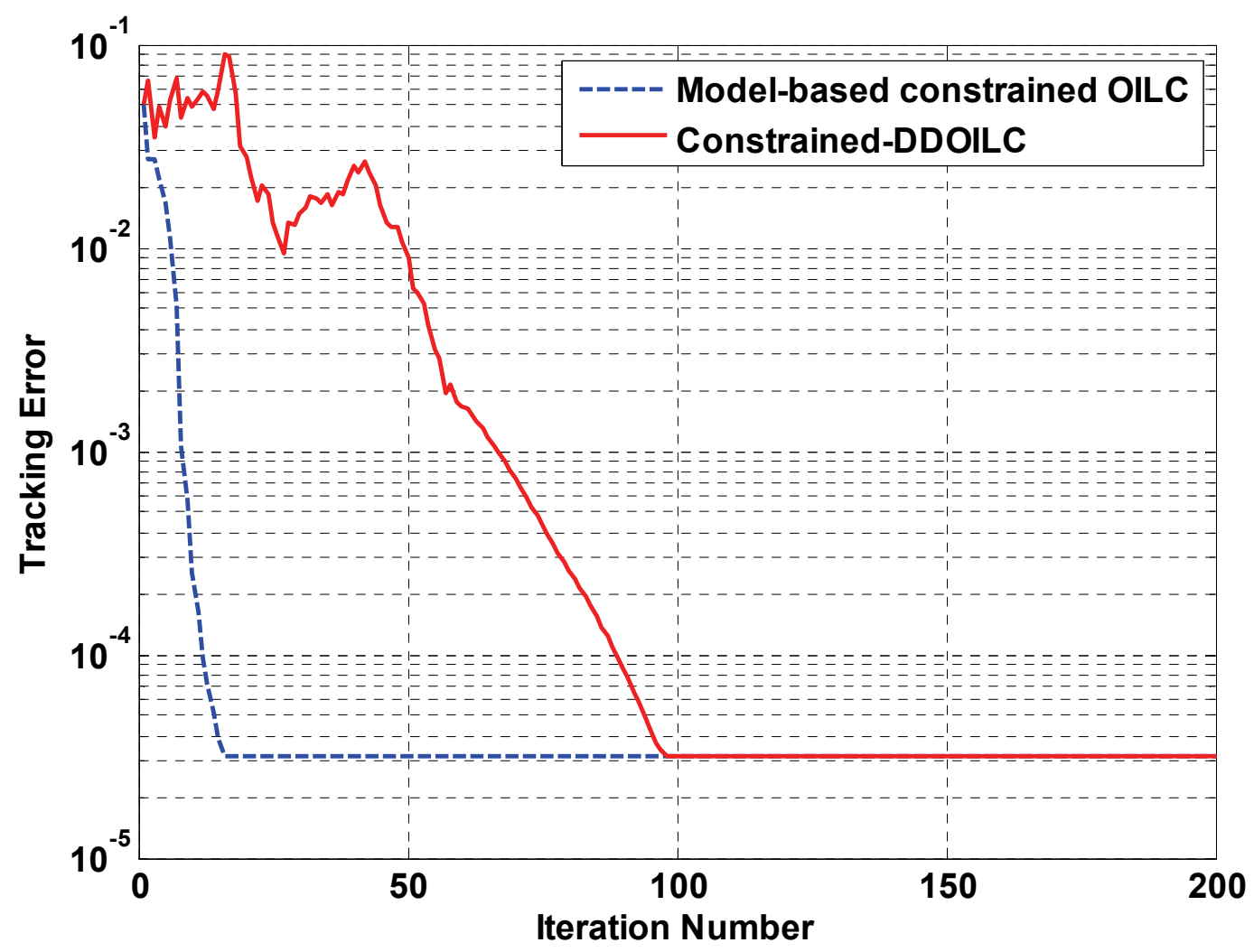

Fig. 23 The error convergence of the model-based constrained OILC and data-based constrained-DDOILC for exactly known linear system 
For the two control methods, the controller parameters are set identically as: $x_{1}(0)=0.1, x_{2}(0)=0$; $u_{0}(t)=0, t \in\{0,1, \cdots, 10\}, \boldsymbol{Q}=\boldsymbol{I}$, and $\boldsymbol{R}=0.5 \boldsymbol{I}$; and the parameters of the estimator in the constrained DDOILC are set as $\eta=0.5, \mu=0.001$, and $\hat{\boldsymbol{\varphi}}_{0}(t)=0.15$. By applying the model-based constrained optimal ILC (50) and the data-based constrained-DDOILC (21) - (22) proposed in this work, the simulation results are shown in Fig. 23. It is verified that the convergence performance by using the model-based constrained OILC, where the exact $\boldsymbol{A}, \boldsymbol{B}, \boldsymbol{C}$ matrices are utilized to calculate the control input, is better than that using the proposed constrained-DDOILC. The latter does not use any information about matrices $\boldsymbol{A}, \boldsymbol{B}$, and $\boldsymbol{C}$.

In practice, an exactly known linear model is hard to obtain and is generally affected by various uncertainties. If there are some large uncertainties in the linear model, the convergence performance of the traditional constrained OILC may be degraded greatly. However, the constrained data-driven OILC does not depend on the linear model and thus can still achieve a satisfactory performance in the presence of large uncertainties to the system model. To simulate this case, the stochastic perturbations to the system matrices $\boldsymbol{A}$ and $\boldsymbol{B}$ are considered. That is $\boldsymbol{A}_{k}(t)=\left[\begin{array}{cc}0+v_{k}(t) & 1+v_{k}(t) \\ -0.5+v_{k}(t) & -0.5+v_{k}(t)\end{array}\right]$ and $\boldsymbol{B}_{k}(t)=\left[\begin{array}{c}0+v_{k}(t) \\ 1+v_{k}(t)\end{array}\right]$ are stochastically time-iteration-varying, where $v_{k}(t)$ varies randomly in the interval of $\left[\begin{array}{ll}-1.5 & 1.5\end{array}\right]$ shown in Fig. 24 .

By applying the model-based constrained OILC and the data-based constrained-DDOILC under the same simulation condition, the simulation results are shown in Fig. 25. It is seen that the data-based constrainedDDOILC is more effective in dealing with the process with strong uncertainties than the model-based constrained OILC because its controller design does not depend on any explicit model information of the controlled plant. On the other hand, the model-based constrained OILC may not guarantee the error convergence any more if a large uncertainty occurs. 


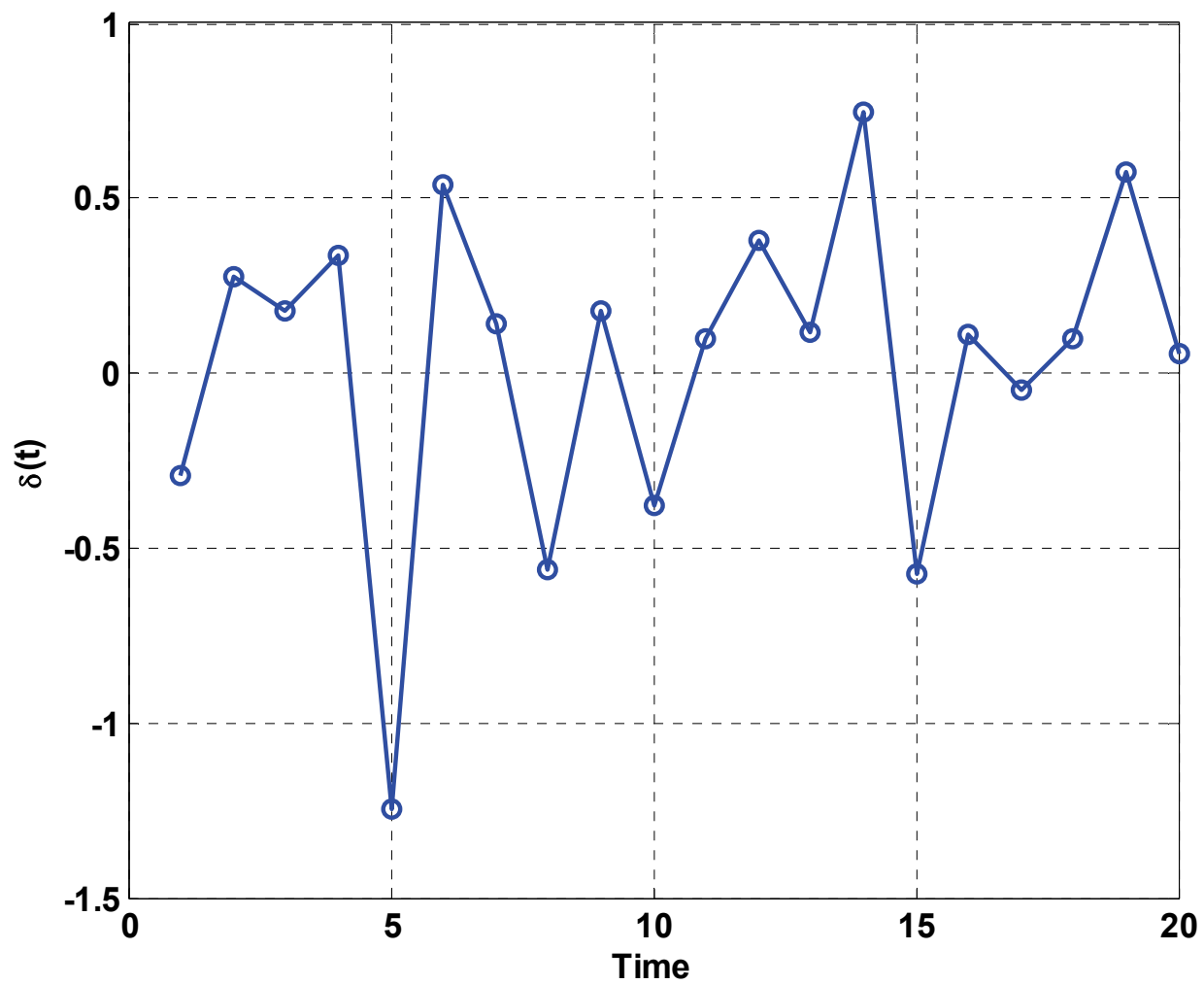

Fig. 24 The random values of $v_{k}(t)$

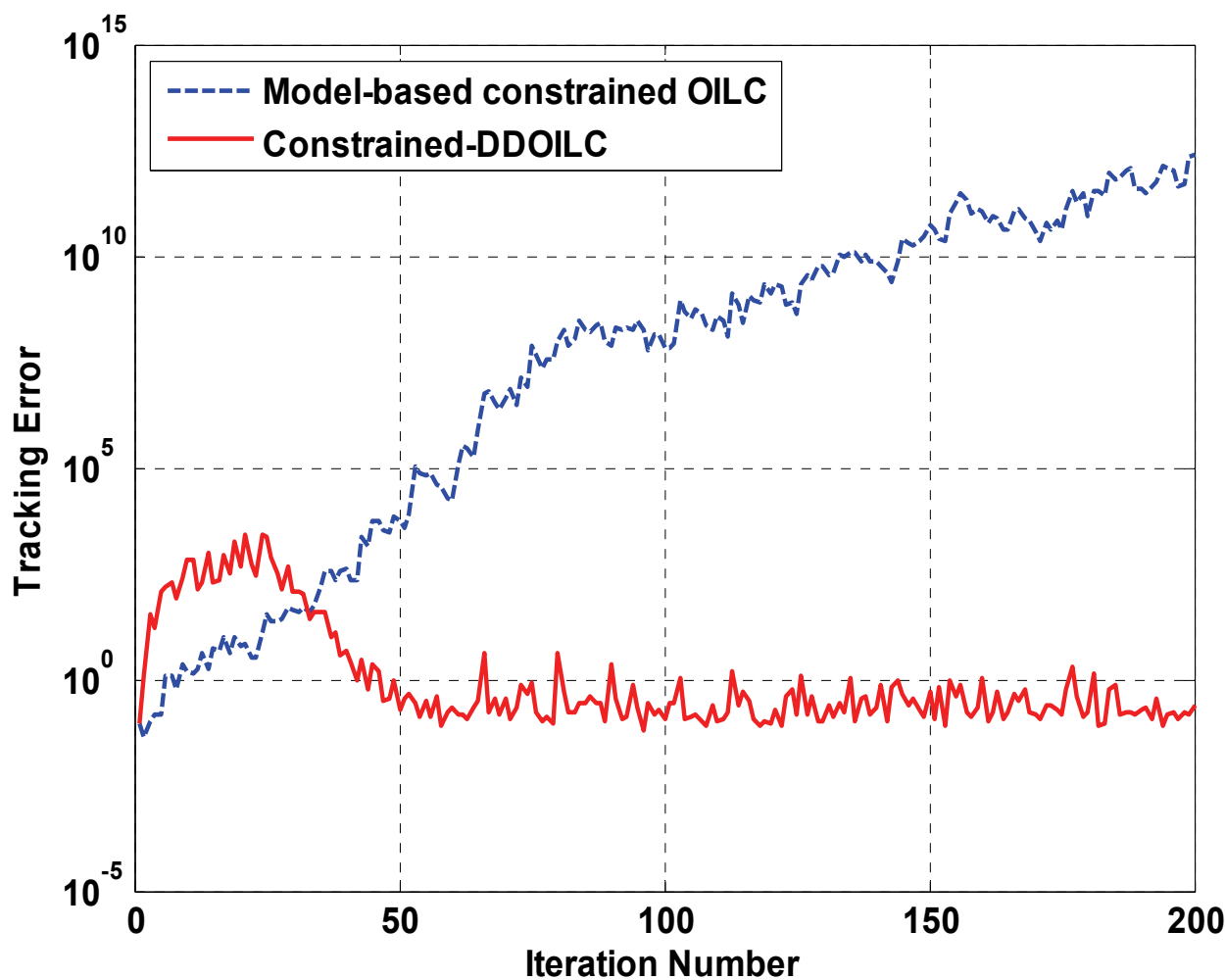

Fig. 25 The error convergence of the model-based constrained OILC and data-based constrained-DDOILC for strongly uncertain linear systems 


\section{Conclusion}

System nonlinearities, uncertainties, and constraints are inevitable in practical applications. By taking a class of nonlinear and non-affine discrete-time systems into consideration, this work proposes a constrained-DDOILC for a traditional trajectory tracking problem and a constrained-DDOPTPILC for a pointto-point control problem where the exact tracking performance is required only at the predesigned points instead of all points of a complete trajectory. The key-point before proceeding to the controller design and analysis is the iterative dynamic linearization method introduced. Then the following derivations depend on the I/O measurements only without the need of explicit model knowledge. One of the major advantages of the proposed approaches is that the optimal learning gain can be updated iteratively to enhance control performance. From the simulation results, one can conclude that: (a) the proposed constrained methods attain a better performance without violations of constraints in the system output or the control input by incorporating the I/O constraints into the controller design; (b) for a point-to-point tracking task, the proposed constrained-DDOPTPILC outperforms the proposed constrained-DDOILC with faster convergence and reduced control effort by removing extra constraints on the unnecessary points; (c) the proposed constrained methods do not depend on the linear model and thus can still achieve a satisfactory performance in the presence of large disturbances to the system model. The robustness of the proposed constrained methods to exogenous uncertainties has been illustrated by taking a realistic nonlinear batch reactor under different levels of stochastic disturbances as an example. Further, the issue of the parameter selection has also been discussed as a guideline for the practical engineering applications.

\section{Acknowledgements}

This work was supported by National Science Foundation of China (61374102, 61433002, \& 61120106009), Taishan Scholar program of Shandong Province of China, and Alberta Innovates Technology Futures (AITF). 


\section{References}

[1] S. Arimoto, S. Kawamura, and F. Miyazaki, Bettering operation of robots by learning, Journal of Robotic Systems, vol. 1, pp. 123-140, 1984.

[2] J.-X. Xu and Y. Tan, Linear and Nonlinear Iterative Learning Control, Springer-Verlag Berlin Heidelberg, 2003.

[3] D. A. Bristow, M. Tharayil, and A. G. Alleyne, A survey of iterative learning control: a learningbased method for high-performance tracking control, IEEE Transactions on Control Systems Magazine, vol. 26, no. 3, pp. 96-114, 2006.

[4] F. J. Doyle, An anti-windup input-output linearization scheme for SISO system, Journal of Process Control, vol. 9, pp. 220-231, 1999.

[5] J.-X. Xu, Y. Tan, T.-H. Lee, Iterative learning control design based on composite energy function with input saturation, Automatica, vol. 40, pp. 1371-1377, 2004.

[6] S. S. Saab, On the P-type learning control, IEEE Transactions on Automatic Control, vol. 39, pp. 2298-2302, 1994.

[7] M. Sun and D. Wang, Anticipatory iterative learning control for nonlinear systems with arbitrary relative degree, IEEE Transactions on Automatic Control, vol. 46, no. 5, 783-788, 2001.

[8] B. J. Driessen and N. Sadegh, Multi-input square iterative learning control with input rate limits and bounds. IEEE Transactions on Systems, Man, and Cybernetics-Part B, vol. 32, no. 4, pp. 545-550, 2002.

[9] B. Chu and D. H. Owens, Iterative learning control for constrained linear systems, International Journal of Control, vol. 83, no.7, pp. 1397-1413, 2010.

[10] Y. Tan, J. Xu, M. Norrlof, C. Freeman, On reference governor in iterative learning control for dynamic systems with input saturation, Automatica, vol. 47, no. 11, pp. 2412-2419, 2011.

[11] Y. Tan, H. Dai, D. Huang, and J.-X. Xu, Unified iterative learning control schemes for nonlinear dynamic systems with nonlinear input uncertainties, Automatica, vol. 48, pp. 3173-3182, 2012 
[12] Moore K L. Iterative learning control: an expository overview//Applied and computational control, signals, and circuits. Birkhäuser Boston, 1999: 151-214.

[13] Amann N., Owens D. H., and Rogers E., Iterative learning control using optimal feedback and feedforward actions. International journal of control, 65(2): 277-293, 1996

[14] Amann N., Owens D. H., and Rogers E., Robustness of norm-optimal iterative learning control. In Proceedings of International Conference on Control, volume 2, pages 1119-1124, Exeter UK, September 1996.

[15] Amann N., Owens D. H., and Rogers E., Iterative learning control for discrete-time systems with exponential rate of convergence. IEE Proceedings - Control Theory and Applications, 143(2): 217-224, 1996.

[16] Lee J H, Lee K S, Kim W C. Model-based iterative learning control with a quadratic criterion for time-varying linear systems. Automatica, 2000, 36(5): 641-657.

[17] S. Mishra, U. Topcu, and M. Tomizuka, Optimization-based constrained iterative learning control', IEEE Transactions on Control Systems Technology, vol. 19, pp. 1613 -1621, 2011.

[18] N. Amann, D.H. Owens, E. Rogers, Predictive optimal iterative learning control, International Journal of Control, vol. 69, no. 2, pp. 203-226, 1998.

[19] K. S. Lee, I. S. Chin, H. J. Lee, and J. H. Lee, Model predictive control technique combined with iterative learning for batch processes, AIChE Journal, vol. 45, no. 10, pp. 2175-2187, 1999.

[20] L. P. Wang, C. T. Freeman, S. Chai, and E. Rogers, Predictive-repetitive control with constraints: From design to implementation, Journal of Process Control, vol. 23, no. 7, pp. 956-967, 2013.

[21] I.-S. Chin, S. J. Qin, K. S. Lee, and M. Cho, A two-stage iterative learning and batch control technique with independent disturbance rejection capability, Automatica, vol. 40, no. 11, pp. 1913-1922, 2004.

[22] T. Liu, and Y. Wang, A synthetic approach for robust constrained iterative learning control of piecewise affine batch processes, Automatica, vol. 48, pp. 2762-2775, 2012. 
[23] R. H. Chi, Z. S. Hou and J.-X. Xu, Adaptive ILC for a class of discrete-time systems with iterationvarying trajectory and random initial condition, Automatica, vol. 44, pp. 2207-2213, 2008.

[24] C. K. Yin, J.-X. Xu, and Z. S. Hou, A High-order Internal model Based Iterative Learning Control Scheme for Nonlinear Systems with Time-Iteration-Varying Parameters, IEEE Transactions on Automatic Control, vol. 55, no. 11, pp. 2665-2670, 2010.

[25] X. D. Li, T. F. Xiao, and H. X. Zheng, Adaptive discrete-time iterative learning control for non-linear multiple input multiple output systems with iteration-varying initial error and reference trajectory, IET Control Theory and Applications, vol. 5, no. 9, pp. 1131-1141, 2011.

[26] Y. Miao, J. Wang, and D. L. Qi, Discrete-time Adaptive Iterative Learning Control for high-order nonlinear systems with Unknown Control Directions, International Journal of Control, vol. 86, no. 2, pp. 299-308, 2013.

[27] J.-X. Xu and J. Xu, Output constraints State-Constrained Iterative Learning Control for a Class Of MIMO Systems, IEEE Transactions on Automatic Control, vol. 58, no. 5, pp. 1322-1327, 2013

[28] J. Xu, and J.-X. Xu, Output constraints Iterative learning control for output-constrained systems with both parametric and nonparametric uncertainties, Automatica, vol. 49, pp. 2508-2516, 2013

[29] Z. S. Hou and Z. Wang, From model-based control to data-driven control: survey, classification and perspective, Information Sciences, vol. 235, pp. 3-35, 2013.

[30] Z. S. Hou and S. T. Jin, Model Free Adaptive Control: Theory and Applications. CRC press, 2013

[31] S. Yin, X. W. Li, H. J. Gao, and O. Kaynak, Data-Based Techniques Focused on Modern Industry: An Overview, IEEE Transactions on Industrial Electronics, 2015, 62(1): 657-667.

[32] Z. Wang, D. R. Liu, Data-based stability analysis of a class of nonlinear discrete-time systems, Information Sciences, vol. 235, pp. 36-44, 2013.

[33] P. Janssens, G. Pipeleers, and J. Swevers, A data-driven constrained norm-optimal iterative learning control framework for LTI systems, IEEE Transactions on Control Systems Technology, vol. 21, no. 2, pp. 546-551, 2013. 
[34] R. H. Chi, D. W. Wang, Z. S. Hou, and S. T. Jin, Data-driven optimal terminal iterative learning control, Journal of Process Control, vol. 22, no. 10, pp. 2026-2037, 2012.

[35] R. H. Chi, Z. S. Hou, B. Huang, and S. T. Jin, A unified data-driven design framework of optimalitybased generalized iterative learning control, Computers and Chemical Engineering, vol. 77, pp. 10$23,2015$.

[36] C. T. Freeman, Z. Cai, E. Rogers, and P. L. Lewin, Iterative learning control for multiple point-topoint tracking application, IEEE Trans. Control Syst. Technol., vol. 19, no. 3, pp. 590-600, 2011

[37] T. D. Son, H.-S. Ahn, K. L. Moore, Iterative learning control in optimal tracking problems with specified data points, Automatica, vol. 49, pp. 1465-1472, 2013.

[38] C.T. Freeman, Constrained point-to-point iterative learning control with experimental verification, IEEE Transactions on Control Engineering Practice, vol. 20, pp. 489-498, 2012

[39] W. H. Ray, Advanced process control, McGraw-Hill, New York, 1981.

[40] J. S. Logsdon, and L. T. Biegler, Accurate solution of differential-algebraic optimization problems, Ind. Eng. Chem. Res., vol. 28, pp. 1628-1639, 1989.

[41] J. S. Logsdon and L. T. Biegler, Decomposition Strategies for Large-scale Dynamic Optimization Problems, Chemical Engineering Science, vol. 47, no. 4, pp. 851-864, 1992. 